\title{
Transition from order to chaos in the wake of an airfoil
}

\author{
By K. WILLIAMS-STUBER† AND M. GHARIB \\ Department of Applied Mechanics and Engineering Sciences, University of California, \\ San Diego, La Jolla, CA 92093, USA
}

(Received 7 June 1988 and in revised form 14 July 1989)

An experimental effort is presented here that examines the nonlinear interaction of multiple frequencies in the forced wake of an airfoil. Wakes with one or two distinct frequencies behave in an ordered manner - being either locked or quasi-periodic. When a third incommensurate frequency is added to the system, the flow demonstrates chaotic behaviour. Previously, the existence of the three-frequency route to chaos has been reported only for closed system flows. It is important to note that this chaotic state is obtained at a low Reynolds number. However, the chaotic flow shows localized characteristics similar to those of high Reynolds number turbulent flows. The degree of chaotic behaviour is verified by applying ideas from nonlinear dynamics (such as Lyapunov exponents and Poincaré sections) to the experimental data, thus relating the basic physics of the system to the concepts of mode interaction and chaos. Significant changes to the vortex configuration in the wake and to the r.m.s. velocity profile occur during the transition from order to chaos.

\section{Introduction}

Many engineering systems can be characterized by the existence of multiple frequencies. The interaction of these frequencies can produce catastrophic results if resonance conditions are established. The mode (frequency) competition is frequently the consequence of nonlinear interactions in the system. When the system contains a sensitive dependence on the initial conditions, the system is said to be 'chaotic'. Chaotic systems have the interesting property that they produce essentially unpredictable behaviour even though the governing equations of motion are deterministic. Such is the case for the phenomenon of turbulence.

In recent years, attempts have been made to explain the transition from laminar to turbulent flow in terms of ideas from the relatively new field of nonlinear dynamics. Many examples of chaotic behaviour have been observed in fluid mechanical systems whose governing equations (the Navier-Stokes equation) are nonlinear owing to the presence of the advection terms. The most extensive documentation of chaotic behaviour in hydrodynamic systems exists for two experiments: Rayleigh-Bénard convection and Taylor-Couette flow which are examples of fully bounded (closed) flows. For an extensive list of references on these flows, see Gollub \& Benson (1980) and Swinney (1983). Closed flows are identified by the fact that a particle in the flow retains a history of its location in the system over all cycles of motion. In an open system (e.g. a water tunnel or channel), the particles

$\dagger$ Present address : Department of Mechanical and Environmental Engineering, University of California, Santa Barbara, CA 93106, USA. 
are redistributed after each cycle. The location of a particle in one cycle is completely uncorrelated with its position in the next cycle. In other words, the velocity profile at the entrance to the open system test section is relatively uninfluenced by downstream events. In addition, closed systems contain significantly less background noise than open systems.

It has been observed that for some conditions the transition to chaotic states and to turbulence in fully bounded (closed) fluid flows occurs according to scenarios generally associated with low-order dynamical systems. The question arises as to whether there is a correspondence between the behaviour of these low-order dynamical systems and the transition to turbulence in open fluid systems such as wakes, shear layers, and boundary layers even though, in theory, these closed fluid mechanical systems have an infinite number of degrees of freedom.

The search for chaotic behaviour in open systems has focused on the wake of a thin cylinder. Sreenivasan (1985) reported the existence of 'windows of chaos' in the laminar wake of a cylinder at certain values of the Reynolds number. The route to chaos used to describe the wake behaviour was the Ruelle-Takens-Newhouse ('RTN') scenario in which two frequencies can exist in a flow in a quasi-periodic state, but the addition of a third frequency results in chaotic motion (Newhouse, Ruelle \& Takens 1978). Sreenivasan attributed the chaotic transition to the flow dynamics in which the Reynolds number was the only control parameter.

Van Atta \& Gharib (1987) demonstrated that the chaotic state observed by Sreenivasan was the result of an aeroelastic coupling between the cylinder vibration modes and the vortex wake. The second frequency in the flow was related to the primary vibration mode of the cylinder. In essence, the vibrating wire acted as a forcing mechanism. An additional control parameter was contributing to the system - the tension on the cylinder which dictates the vibration modes - resulting in the chaotic behaviour observed by Sreenivasan.

Owing to the coupled nature of the wire/wake system, the amplitude and frequency of forcing could not be independently controlled by the investigators. Presented here is a series of fully controllable experiments in which the interaction of multiple independent frequencies in an open flow (the wake of an airfoil) is examined. Various behaviours associated with the RTN route to chaos are observed. Standard diagnostics from nonlinear dynamics (Poincaré sections and Lyapunov exponents) are applied to the experimental data to establish the dynamical nature of the system. In addition, the flows are also examined from a classical fluid dynamics point of view (flow visualization, velocity and drag measurements, wavenumber spectra) to show that a connection exists between laminar chaotic flows and fully developed turbulent flows.

\section{Experimental procedures}

The experimental apparatus and operational procedures in this paper are the same as reported in Stuber (1988) and Gharib \& Williams-Stuber (1989, hereinafter referred to as I). Perturbations (waves) are introduced to the airfoil boundary layers through the use of the strip heater technique (figure 1). The waves are amplified by the boundary layers and introduced to the wake. The receptivity of this complex system to single frequencies (as determined in I) is presented in figure 2 . This figure shows the response amplitude of a forced frequency as measured in the wake. The most amplified frequency $(4.2 \mathrm{~Hz})$ is close to the natural vortex shedding frequency $(4.075 \mathrm{~Hz})$. In $\mathrm{I}$, it was observed that the flow locks to the forcing frequency over a 


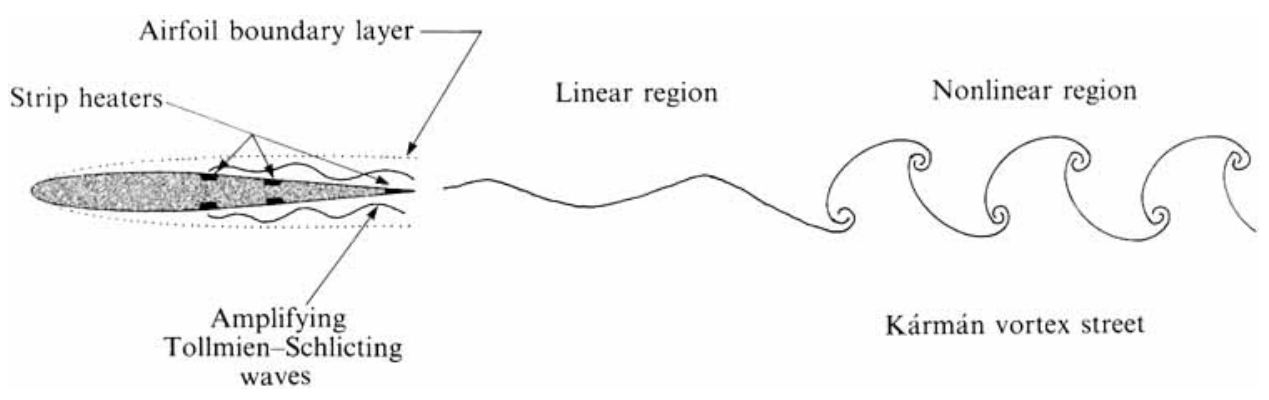

Figure 1. The strip heater technique for introducing waves into the wake of an airfoil.

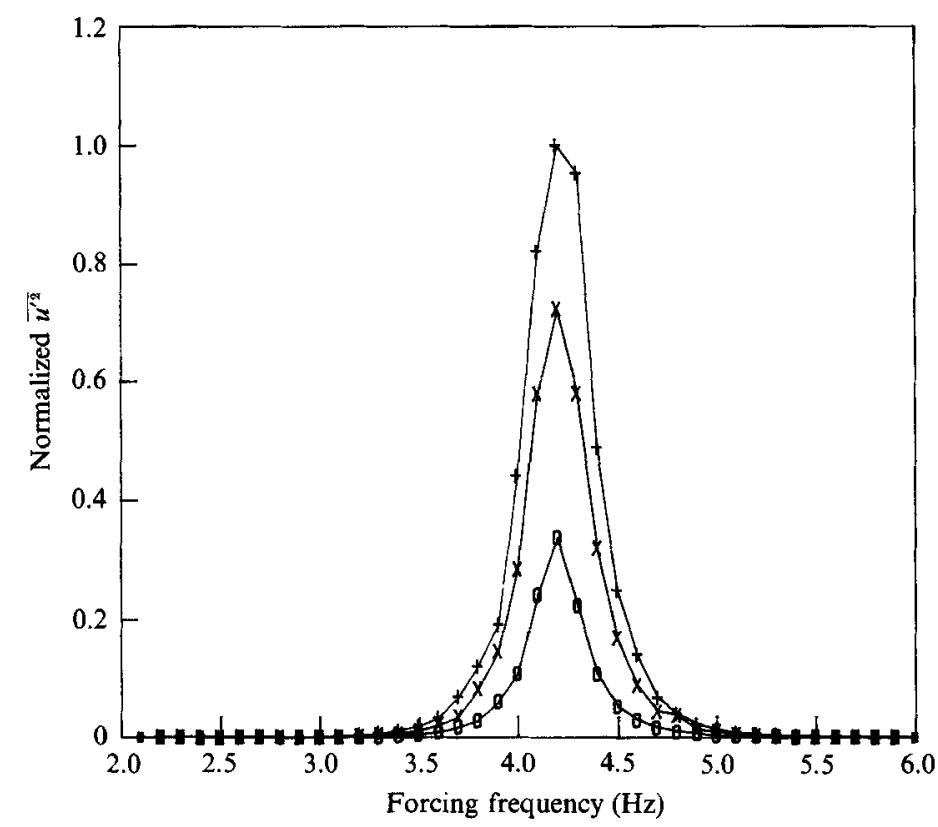

Figure 2. Frequency response curve for three input power levels: $0,5.4 \mathrm{~W} ; \times, 9.8 \mathrm{~W} ;+$, 14.1 W (at $x / c=3$ and $y=\delta$ ).

range of frequencies near the most amplified frequency. The locking range as a function of input power to the strip is shown in figure 3 . Owing to the quadratic joule heating effect, each frequency must be input on a separate strip. Thus, in order to introduce multiple frequencies to the system, additional independent strip heaters were used.

The origin of the coordinate system for the analysis was located at the trailing edge of the airfoil with $y$ in the cross-stream direction and $x$ in the downstream direction. Velocity measurements were performed in the wake at $x / c=3$, where $c$ is the airfoil chord length $(3.0 \mathrm{in}$.$) . This measuring station was selected so that the nonlinear$ evolution and interaction of fluctuations in the wake could be observed. The freestream velocity $\left(U_{\infty}\right)$ was set at $11.5 \mathrm{~cm} / \mathrm{s}$. The point in the wake where the velocity was equal to $99 \%$ of $U_{\infty}$ was designated as $y=\delta$. The Reynolds number based on $\delta\left(R e_{\delta}\right)$ as measured at $x / c=1$ is 590 . When referring to the velocity fluctuations, $\left(u^{\prime 2}\right)^{\frac{2}{2}}$ will designate the r.m.s. velocity and $\overline{u^{\prime 2}}$ will denote the time average of the fluctuation value squared. 


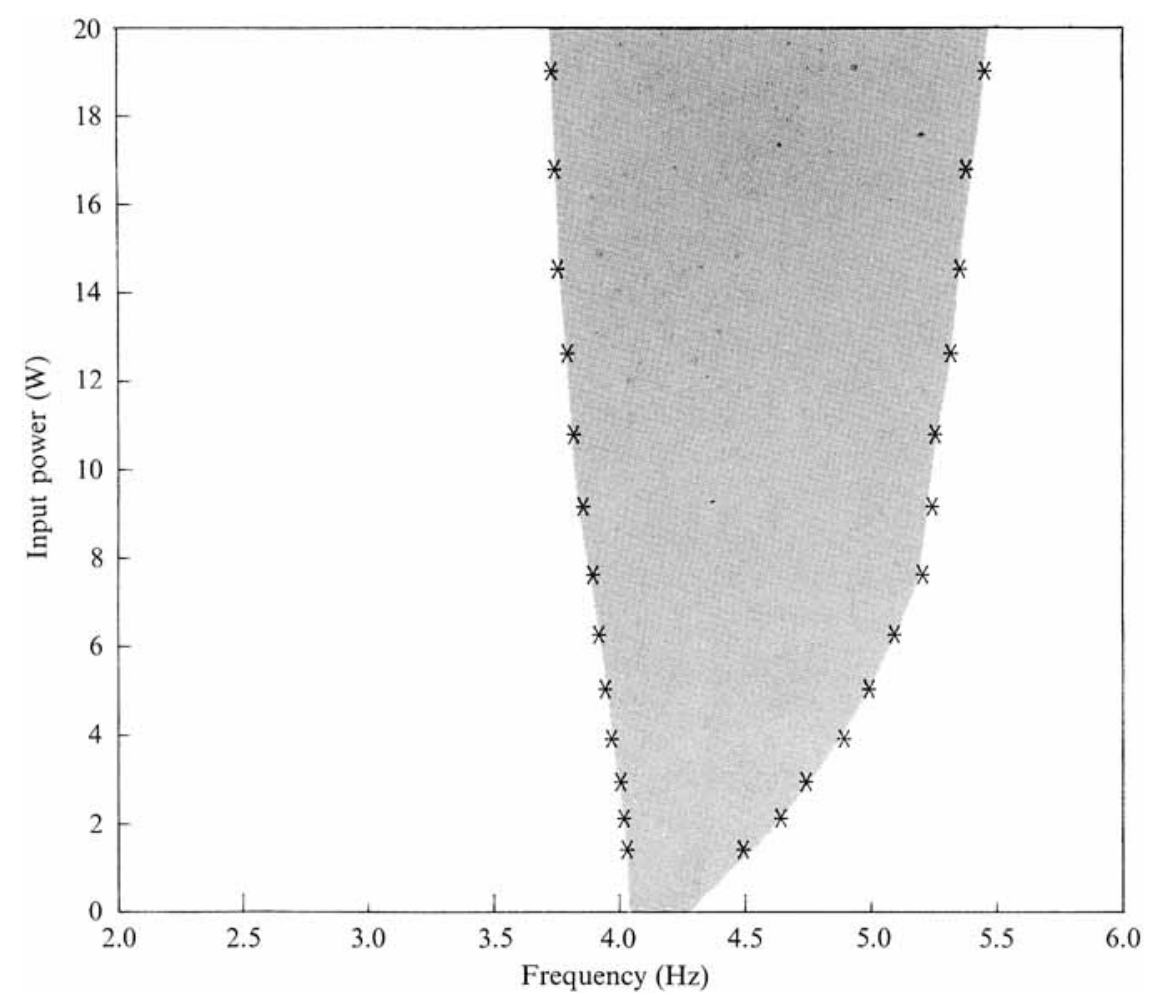

Fraure 3. The locking range (at $x / c=3$ and $y=\delta$ ).

\section{Results and discussion}

\subsection{Flow mapping}

Before proceeding to the flow mapping, it should be noted that power spectrum for the unforced wake is characterized by broad bands around the natural peak and its harmonics (figure 4). This broadness is attributed to jitter in the stagnation point of the airfoil as influenced by background noise in the system. It is noted that the behaviour observed in the wake is due to the introduction of the forced modes because the free-stream noise can be considered constant at a given velocity. In order to establish a cleaner initial state, the natural frequency will be forced continuously at a low level in order to lock the flow to a single frequency.

As in $I$, the first step to be performed for the multi-frequency system is to establish the response of the system. In the previous cases of single-frequency forcing, the main parameters varied were the amplitude and frequency of forcing. By fixing one of the parameters (e.g. the amplitude), the effect of the second parameter was determined by sweeping it over a range of values. This type of procedure led to the results of the receptivity and locking ranges.

With the addition of a second frequency, the behaviour of the system becomes increasingly complex as the two frequencies interact. In order to determine the nature of the interactions, a flow mapping was performed which examined a multitude of combinations of the two frequencies. The measurements were made at $y=\delta$ and at $x / c=3$ so that the nonlinear interactions had time to develop. 

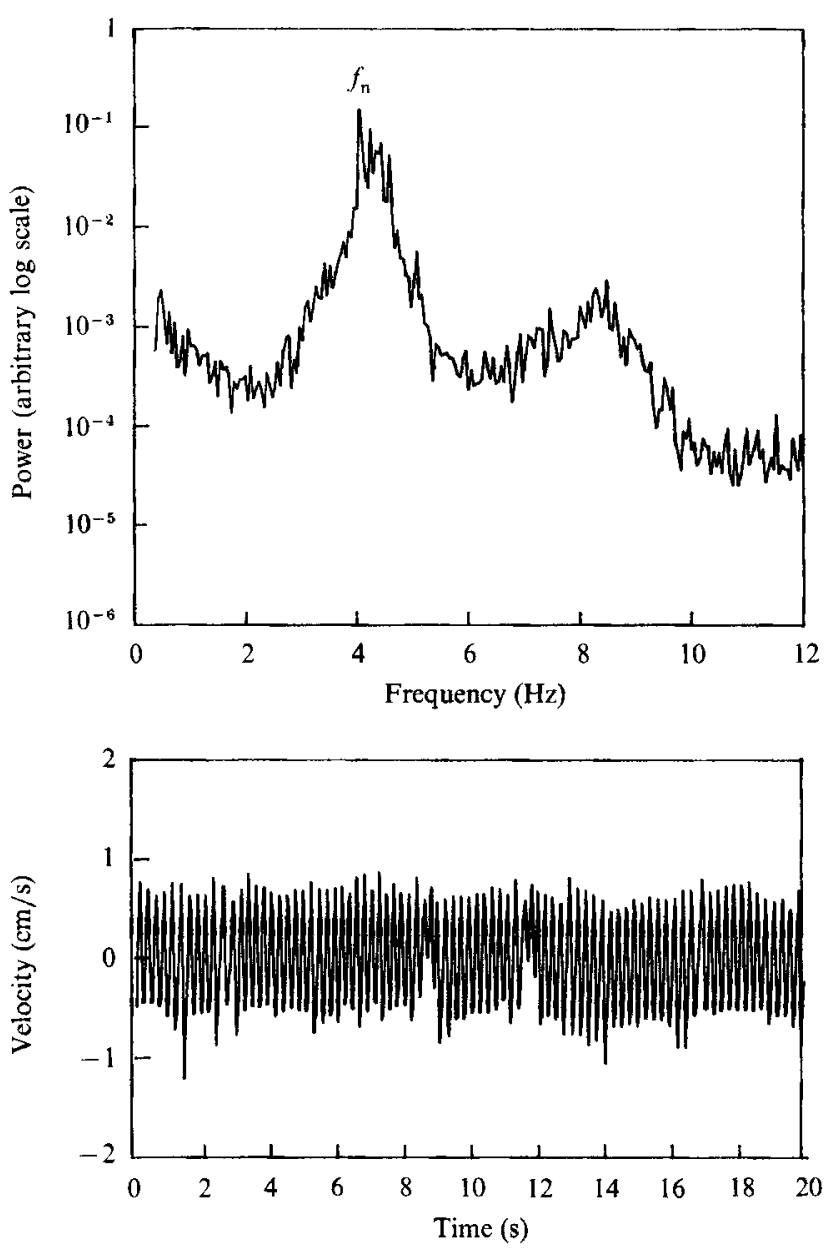

Figure 4. Power spectrum and time series for the natural case (at $x / c=3$ and $y=\delta$ ).

(Arbitrary scale is consistent for all power spectra.)

The procedure for the mapping is outlined below :

(i) $f_{\text {top }}=f_{1}$ was set at one of six reference frequencies $\left(3.5,3.76,4.075 \mathrm{~Hz}=f_{\mathrm{n}}, 4.4\right.$, 5.0 , and $5.5 \mathrm{~Hz}$ ).

(ii) $f_{\text {bottom }}=f_{2}$ was ranged over the values of $3.4-6.0 \mathrm{~Hz}$ in increments of $0.1 \mathrm{~Hz}$.

(iii) The responses at $f_{1}$ and $f_{2}$ in the presence of each other were determined by measuring the bandwidth $\overline{u^{\prime 2}}$ at $f_{1}$ and $f_{2}$ respectively (i.e. the area under the power spectrum in a $0.1 \mathrm{~Hz}$ band centred at the forcing frequency).

Two typical response curves are shown in figure 5. The locking range as defined in I is indicated by the grey band. Depending on the position of $f_{1}$ relative to the locking range of the flow, two transition patterns were observed as $f_{2}$ was swept from 3.4 to $6.0 \mathrm{~Hz}$. First, when $f_{1}$ is fixed in the locking range (figure $5 a: f_{1}=4.075 \mathrm{~Hz}$ ), the response amplitude of $f_{1}$ dominates the $f_{2}$ response amplitude when $f_{2}$ is outside of the locking range. For this forcing configuration, the vortex shedding frequency locks to the $f_{1}$ frequency. The power spectrum of such a case (figure $6: f_{2}=3.5 \mathrm{~Hz}$ ) shows a sharp peak at $f_{1}$ with a much smaller peak at $f_{2}$ (which is indistinguishable from the background). The velocity times series is very uniform. In essence, this first flow 

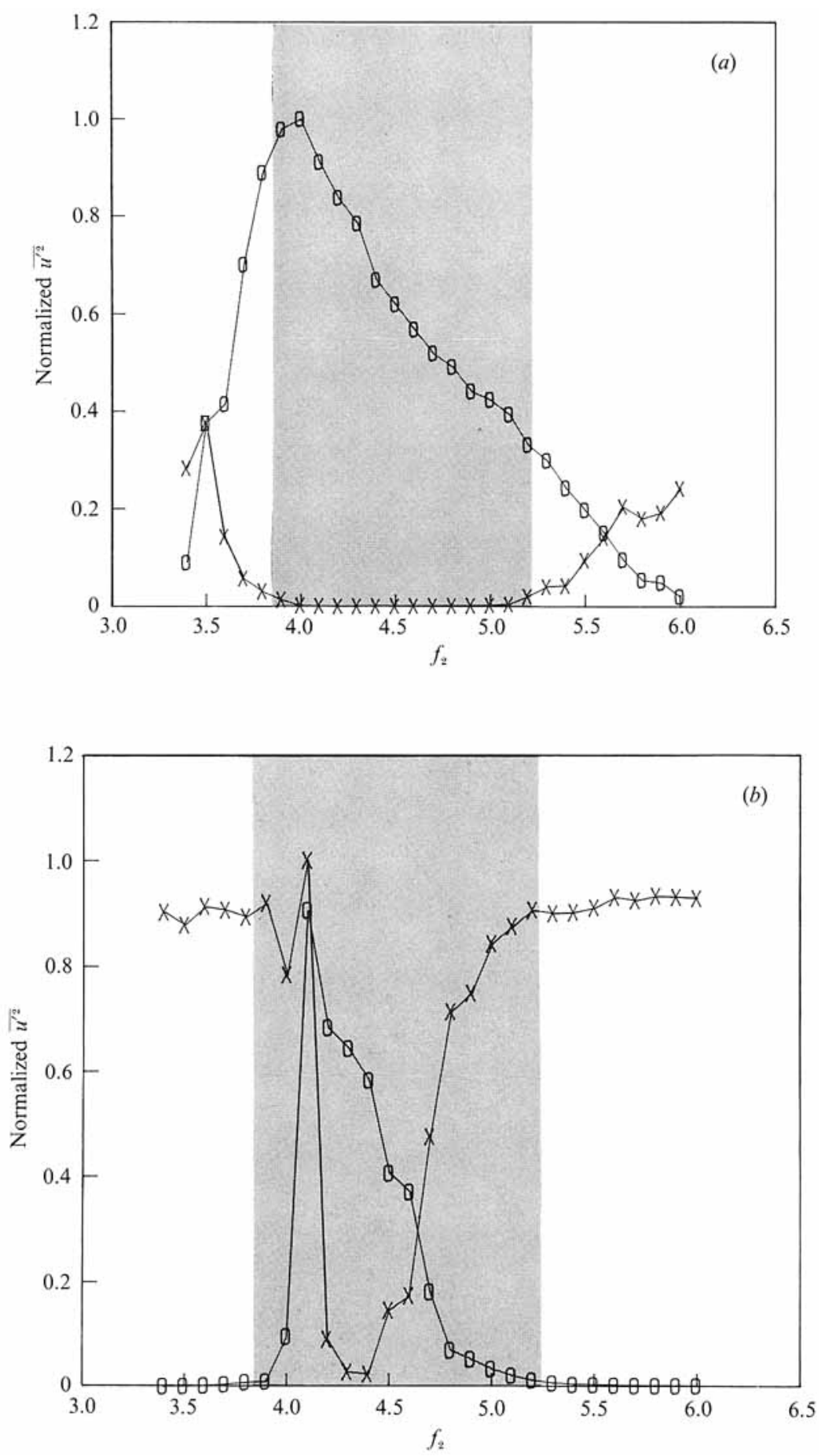

FIGURE 5. Response curves for $f_{1}(x)$ and $f_{2}(0)$ in the presence of each other: $(a) f_{1}$ is fixed in the locking range at $4.075 \mathrm{~Hz} ;(b) f_{1}$ is fixed out of the locking range at $3.5 \mathrm{~Hz}$. 

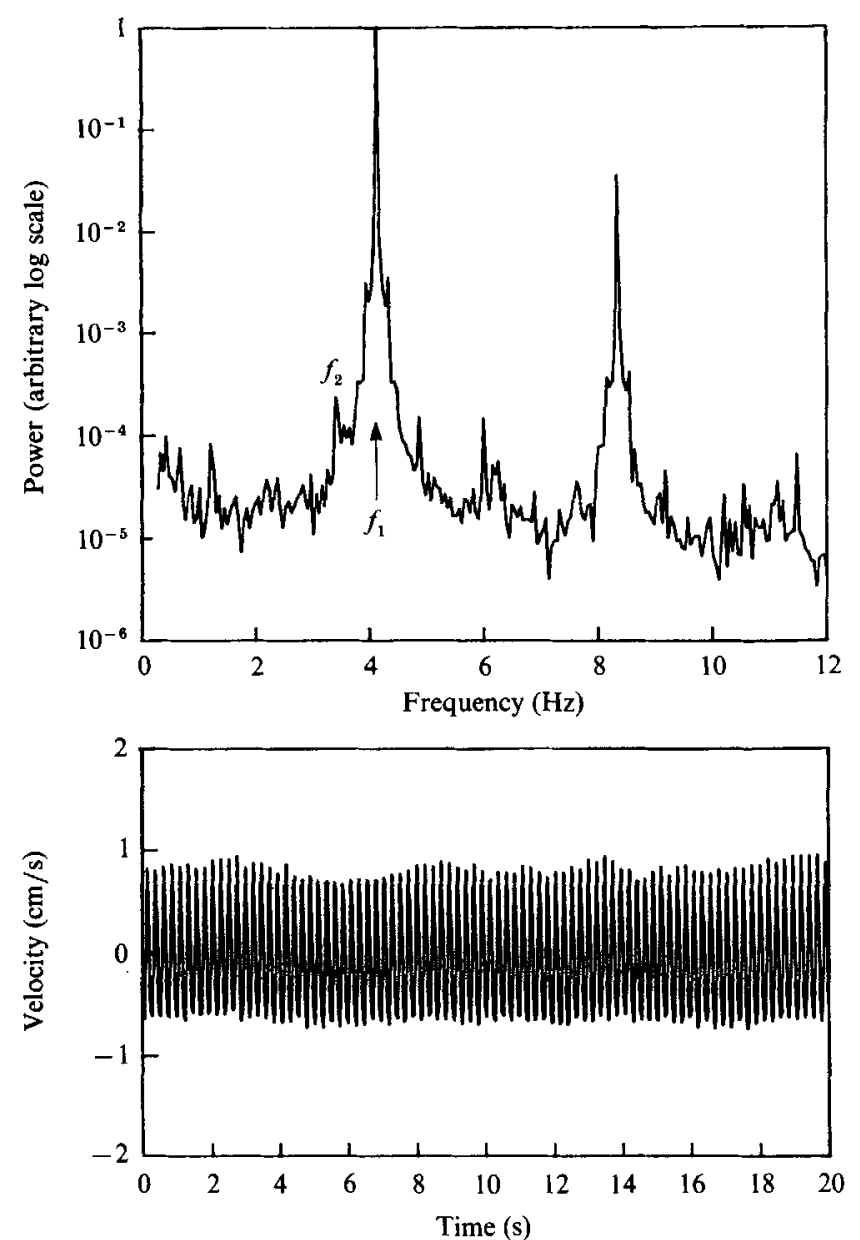

Figure 6. Power spectrum and time series for the locked case (at $x / c=\mathbf{3}$ and $y=\delta$ ).

represents a single-frequency forced system. It is noted that the noise level is significantly decreased compared to the natural flow spectrum. When $f_{2}$ and $f_{1}$ are of equal strength (i.e. both are in the locking range), interactions between the two occur, and a quasi-periodic behaviour is observed. A typical power spectrum (figure $7: f_{2}=4.7 \mathrm{~Hz}$ ) contains peaks at $f_{1}$ and $f_{2}$ as well as peaks at linear combinations of $f_{1}$ and $f_{2}$. The time series contains a strong beating associated with the difference between the two frequencies.

The second pattern occurs when $f_{1}$ is fixed outside of the locking range (figure $5 b$ : $f_{1}=3.5 \mathrm{~Hz}$ ): the response curve shows that the response amplitude of $f_{2}$ dominates much of the range of frequencies. More complex behaviour is observed. Clearly when $f_{2}$ is in the locking range, the flow locks to the forcing frequency as described above. If $f_{2}$ is near the edges of the locking range, quasi-periodic interactions occur which are not as strong as in the first quasi-periodic case. A representative power spectrum (figure $8: f_{2}=4.9 \mathrm{~Hz}$ ) shows only a couple of interaction peaks in addition to peaks at the two forcing frequencies. A small, but noticeable, increase in the noise level is observed, and the natural frequency is starting to reappear. The time series, although somewhat irregular, contains a beating corresponding to the difference between the 

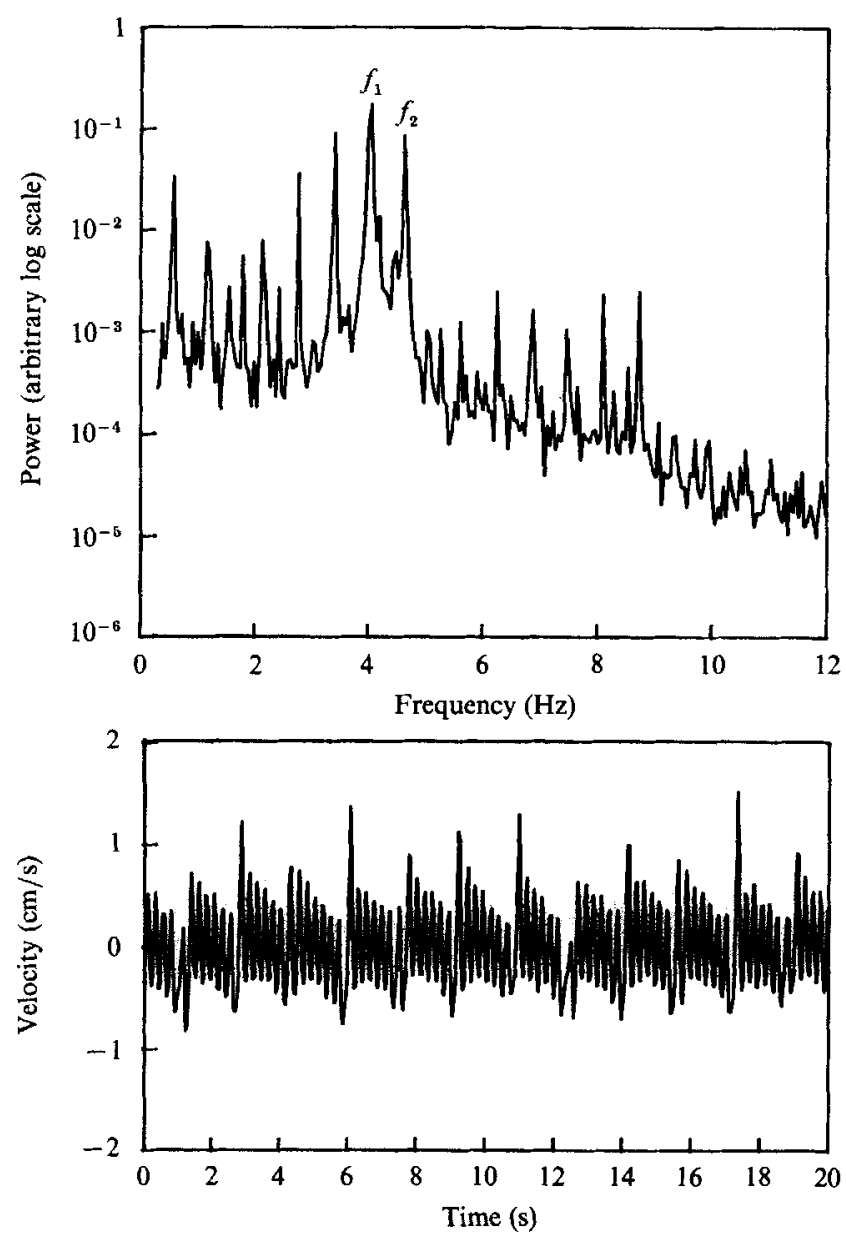

Figure 7. Power spectrum and time series for the strongly quasi-periodic case (at $x / c=3$ and $y=\delta$ ).

forcing frequencies. When neither $f_{1}$ nor $f_{2}$ is in the locking range, the natural frequency can be amplified in the wake. For such a configuration, the power spectrum (figure $9: f_{2}=5.42 \mathrm{~Hz}$ ) is very noisy with peaks only at the two forcing frequencies, the natural frequency, and some of the linear combinations of the three frequencies. The time series is very irregular with no obvious periodicity. The presence of a noisy power spectrum (the base noise level being almost two orders of magnitude greater than in the locked case) with three primary frequencies in the flow $\left(f_{1}, f_{2}\right.$ and $\left.f_{\mathrm{n}}\right)$ suggests a three-frequency chaotic regime according to the RTN scenario.

A schematic summary of the flow behaviour for a two-frequency forced system (in addition to the natural frequency which is forced at a low level) is presented in figure 10. The boundaries and widths of the regions are determined by the relative input amplitudes of the forcing frequencies. The regions are defined as follows:

I Locked to $f_{1}$

II Locked to $f_{2}$

III Quasi-periodic interactions between $f_{1}$ and $f_{2}$ 

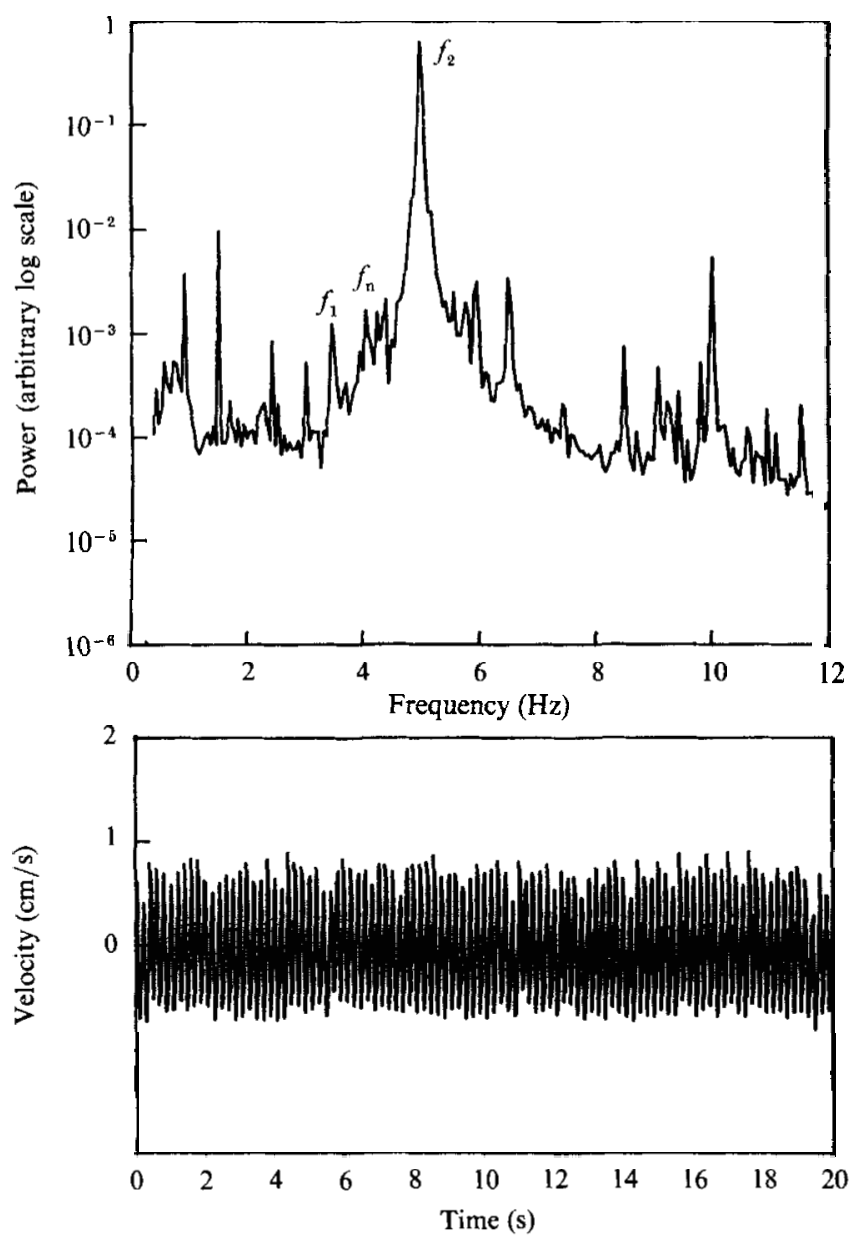

Figure 8. Power spectrum and time series for the weakly quasi-periodic case (at $x / c=3$ and $y=\delta$ ).

IV Possible quasi-periodic interactions between $f_{1}$ and $f_{n}$ or between $f_{2}$ and $f_{n}$. (This region was not fully explored. Based on the results of single-frequency forcing in $\mathrm{I}$, it is most likely that $f_{\mathrm{n}}$ dominates.)

$\mathrm{V}$ Locked to $f_{\mathrm{n}}$ (i.e. $f_{1}$ and $f_{2}$ have absolutely no effect.)

VI Chaotic, interaction between $f_{1}, f_{2}$, and $f_{n}$.

Along the $45^{\circ}$ line $\left(f_{1}=f_{2}\right)$ the system behaves as a single-frequency forced system.

\subsection{Three-frequency route to chaos}

In the final chaotic case noted above, the ratios of the three frequencies $f_{1}: f_{2}: f_{\mathrm{n}}$ is $1: 1.54857: 1.16429$ which is not close to any integer ratios, nor can the three frequencies be obtained by forming linear combinations of each other. The latter observation was determined by considering the sum $S=m_{1} f_{1}+m_{2} f_{2}+m_{3} f_{3}$ where the $m_{i}$ were ranged from -15 to +15 . For all intents and purposes, the three frequencies are incommensurate, based on the above remarks. Using the power spectrum as a primary diagnostic, it would appear that the three-frequency case behaves chaotically. However, owing to the lack of phase information from the 

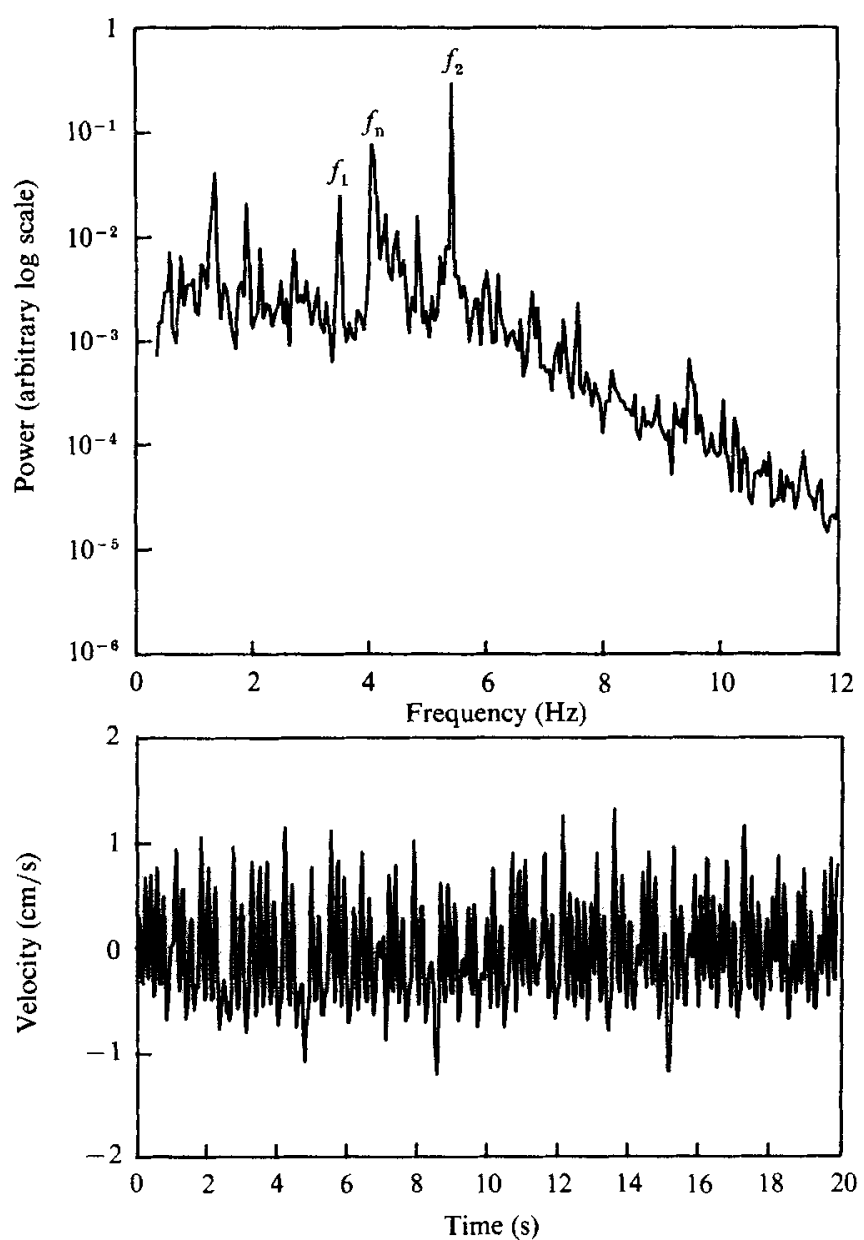

Figure 9. Power spectrum and time series for the chaotic case (at $x / c=3$ and $y=\delta$ ).

power spectrum, further diagnostics from the field of nonlinear dynamies must be applied to the data before a definitive classification is made. A few of the tools used to quantify chaotic behaviour will be presented in the next section. For a more complete description of dimensions and measures of chaos, the reader is referred to Farmer, Ott \& Yorke (1983) and to Eckmann \& Ruelle (1985).

\subsubsection{The nonlinear dynamical analysis of experimental data}

Phase space reconstruction. The first step in analysing experimental data obtained from a dynamical system is to construct the phase space of the system. Given a time series of a single quantity, $U(t)$ (in the current experiment, the $x$-component of velocity), a reconstruction of the phase space can be obtained by using a time delay technique (Packard et al. 1980; Takens 1981) so that

$$
X(t)=\{U(t), U(t+\tau), \ldots, U(t+(m-1) \tau)\},
$$

where $\tau$ is the time delay and $m$ is the embedding dimension (or anticipated number of variables needed to characterize the motion of the system). For the current experiment, an embedding dimension of 5 was selected (which, it was felt, would be 


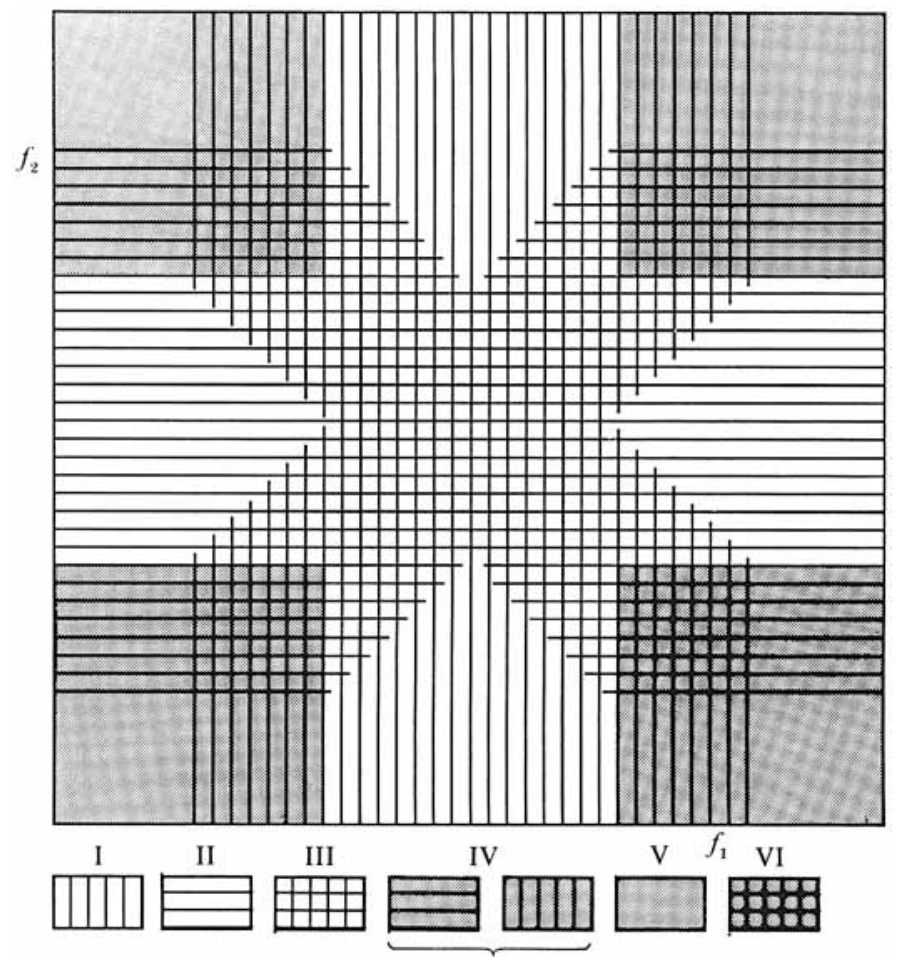

Figure 10. Flow mapping of the interactions of $f_{1}$ and $f_{2}$ in the presence of natural frequency $f_{\mathrm{n}}$ which is forced at a low level. Regions I-VI are defined in the text.

more than sufficient for the locked and quasi-periodic cases, but not necessarily enough for the chaotic case). Although any time delay may be selected, it is preferable to select one corresponding to $\frac{1}{2}-1 \frac{1}{2}$ orbital periods. For the present work, a time delay of 42 points $(0.17 \mathrm{~s}=0.67$ orbital period) was utilized.

The phase space reconstructions (phase portraits) for the natural (unforced) wake and the locked and chaotic cases are presented in figure $11(a-c)$. In each figure, the phase portrait is generated from a time trace at $y=\delta$. Owing to constraints of the graphics system, only the first 5000 points are presented here. However, the nature of the trajectories for each condition did not change when the plots with 22500 points were viewed on a higher level graphics system. The coordinates for the axes are the binary values obtained from the data acquisition system. The value 900 corresponds roughly to $9 \mathrm{~cm} / \mathrm{s}, 1300$ to $13 \mathrm{~cm} / \mathrm{s}$, and so on. In the transition from natural to locked flow (figure $11 a$ to $11 b$ ), the organizing of the phase portrait is evident. Random noise is suppressed in the locked case as was seen previously in the power spectrum. The locked portrait resembles a thin cord or torus. In contrast, the chaotic phase portrait (figure $11 c$ ) resembles tangled balls of yarn rather than thin ribbons.

Poincaré sections. Once the phase space reconstruction is complete, the intersection of trajectories (in the first three dimensions) with a plane can be examined. The set of points intersecting the plane constitutes a Poincaré section of the system. The Poincaré sections provide a better understanding of an additional dimension and possibly structure of the system. For the present work, the intersection of trajectories with a plane at $45^{\circ}$ from the horizontal is presented. Additional Poincaré sections were examined at planes parallel to the $U(t)$ and $U(t+\tau)$ axes; the features were similar to the $45^{\circ}$ plane results. 

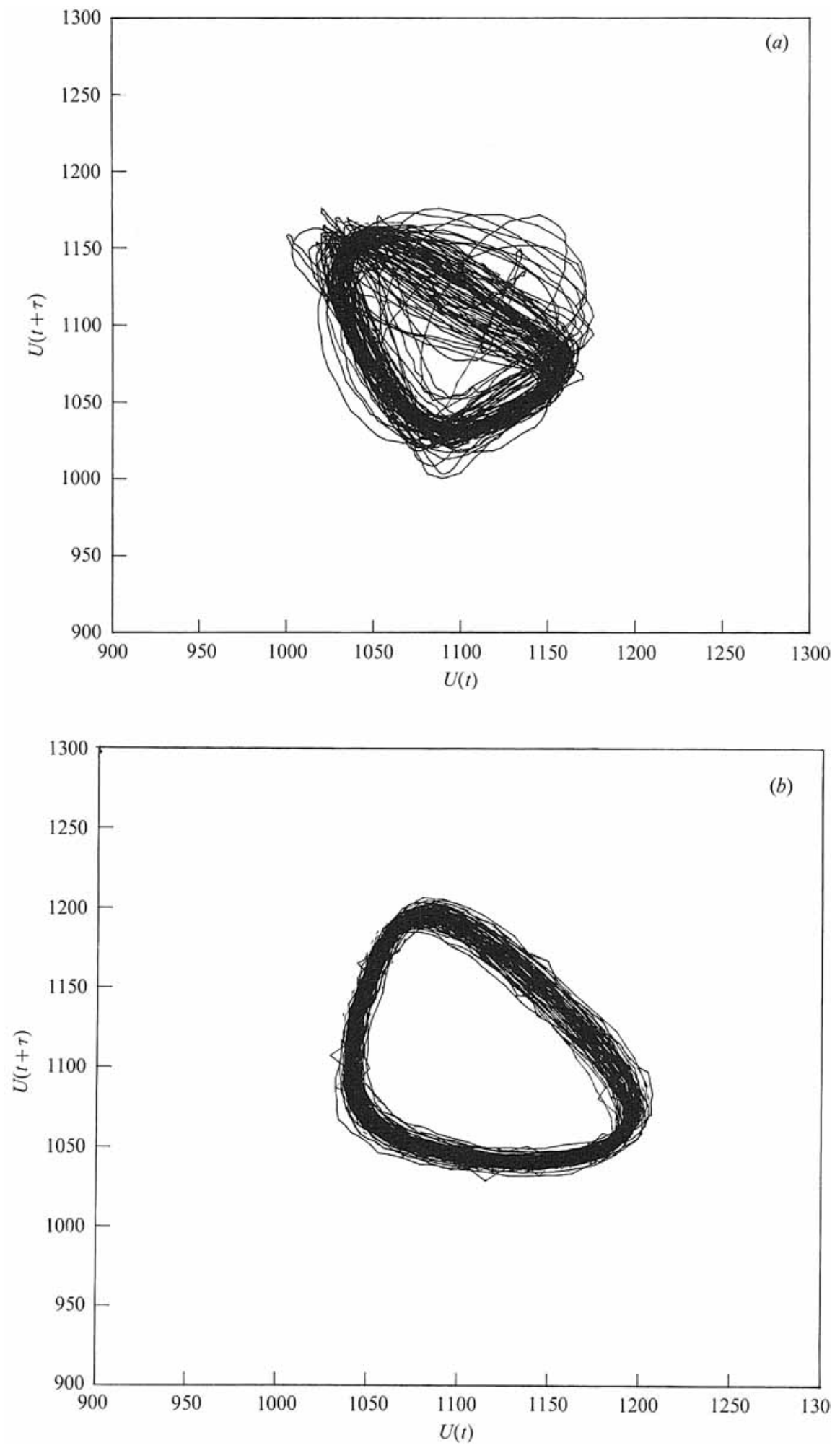

Fraure $11(a, b)$. For caption see facing page. 


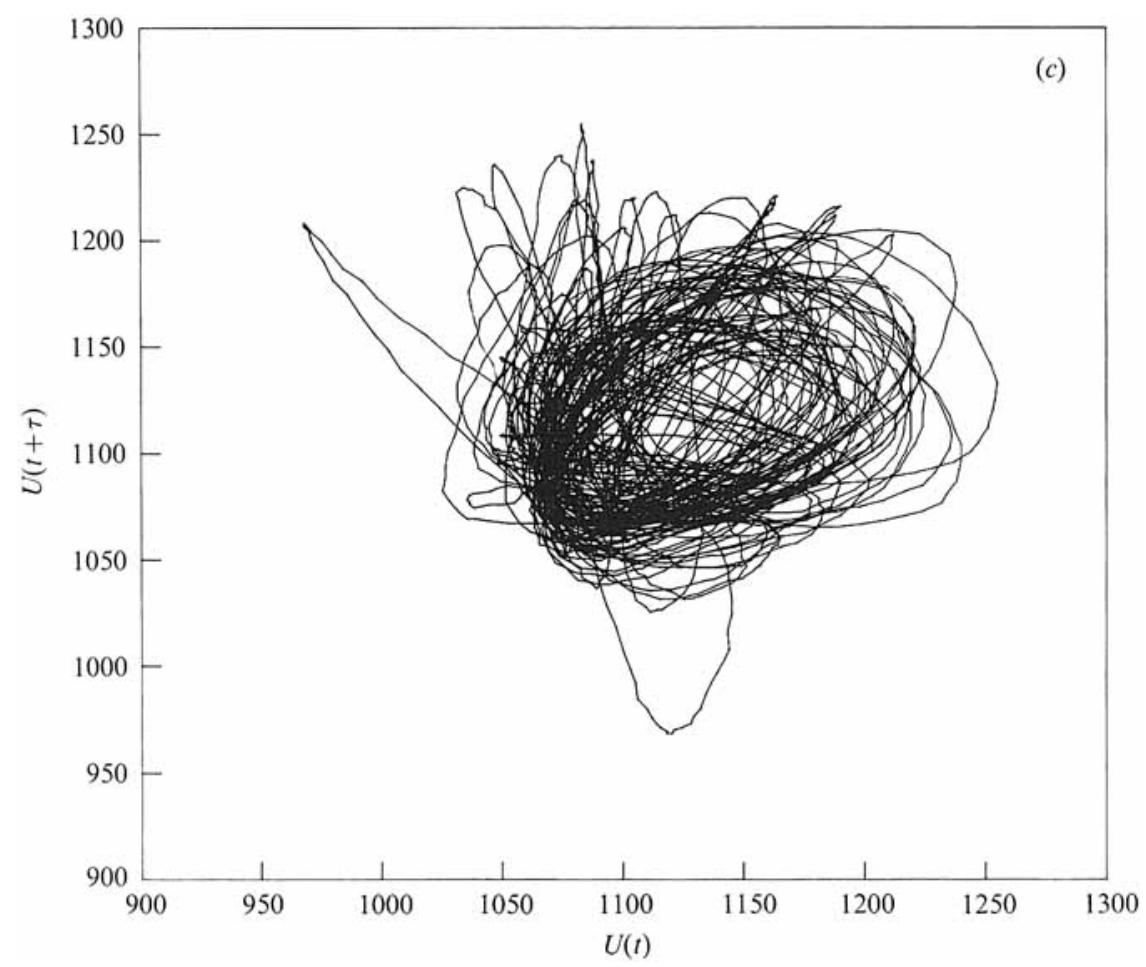

Considering only the two extremes, the Poincaré sections for the locked case and the chaotic case are presented in figures $12(a)$ and $12(b)$, respectively. The intersections shown are from all of the data points in the time series. The Poincaré section of the locked case is characterized by concentrations of points at the intersection of the torus with the plane. The intersections of the chaotic case are randomly scattered across the plane.

Lyapunov exponents. The phase space reconstruction and Poincaré sections provide information about the geometrical nature of the system. To complement those results, quantitative details can be obtained from the reconstructed phase space by measuring the spectrum of Lyapunov exponents of the system (originally defined by Oseledec 1968). Lyapunov exponents quantify the sensitivity of a dynamical system to initial conditions. By considering the largest exponent, $\lambda_{1}$, the dynamical nature of the attractor can be determined. If $\lambda_{1}<0$, all initial conditions eventually converge to the same point, a fixed point. $\lambda_{1}=0$ implies that the length of the principal axis (in phase space) remains constant. This corresponds to a limit cycle (either periodic or quasi-periodic motion). If $\lambda_{1}>0$, the length of the principal axis is diverging (at an exponential rate). When such a condition exists, the attractor is said to be 'strange' or 'chaotic'. The value of the largest Lyapunov exponent for the experimental time series is determined according to an algorithm presented by $W$ olf et al. (1985).

To quantify the nature of the flows as a function of position across the wake, the largest Lyapunov exponent was measured at several points across the wake to form a 'Lyapunov profile'. In presenting the profiles, a three-point average was performed to smooth out fluctuations. 

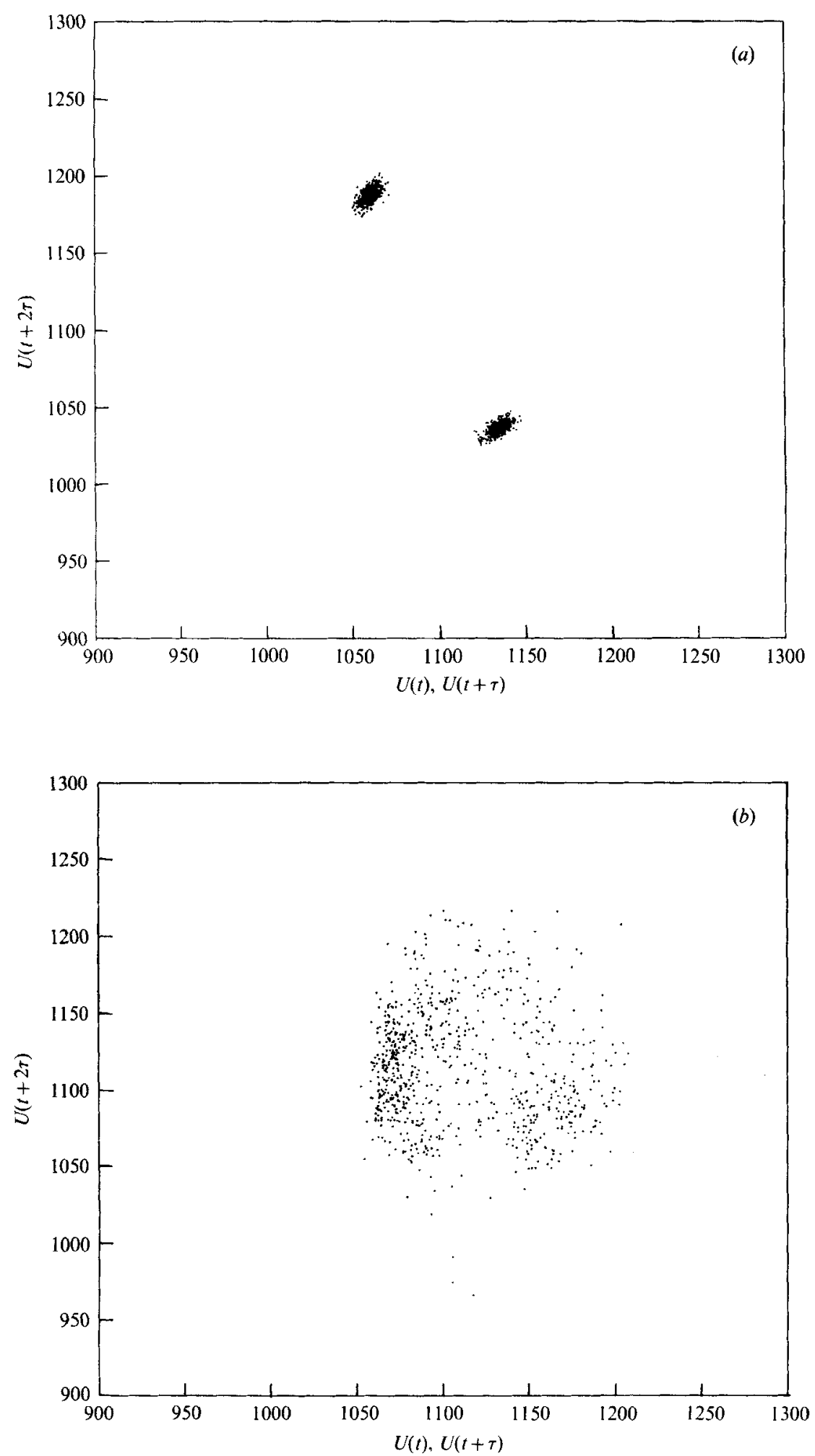

Figure 12. Poincaré section for ( $a$ ) the locked case, $(b)$ the chaotic case (at $x / c=3$ and $y=\delta$ ). 
The profile for the natural (unforced) case (figure $13 a$ ) reveals a distinct variation across the wake. Two peaks exist corresponding to the maxima in the $\left(u^{\prime 2}\right)^{\frac{1}{2}}$ profile. This initial observation seems to imply that the Lyapunov exponent is sensitive to the amplitude of fluctuations in the wake. However, when the flow is locked to the natural frequency (figure $13 b$ ) the peaks observed in the natural profile disappear. The larger amplitude of the natural flow compared to the forced flow can be attributed to the fact that the position of the stagnation point at the leading edge is sensitive to free-stream noise. Hence, the initial condition of the boundary layers varies in a random function which results in a jitter of the vortex motion and, thus, in random fluctuations in the velocity time series. When the flow is forced, a fixed reference for the nature and intensity of perturbations introduced to the wake is established. The forcing actually adds order to the system creating uniform dynamical behaviour across the entire flow. The increase of the exponents at the edges of the profiles is attributed to the effect of free-stream noise.

A comparison of the locked and chaotic cases as well as two quasi-periodic cases (figure $13 \mathrm{c}$ ) demonstrates that the dynamical behaviour of the chaotic case is indeed different than the three ordered cases. The chaotic exponents are typically three times greater than the exponents for the ordered cases. The fact that the maximum Lyapunov exponent for the chaotic case is less than for the natural case is attributed to the relative order created by the presence of forcing.

\subsubsection{Summary of nonlinear dynamics diagnostics}

Through the use of phase portraits, Poincaré sections, and Lyapunov exponents, a distinct transition is seen from ordered to chaotic flow. The results do not indicate a clear distinction within the ordered cases, in particular between the quasi-periodic cases. Researchers familiar with behaviour of closed systems (e.g. Rayleigh-Bénard convection or Taylor-Couette flow) would notice that the values of the ordered case Lyapunov exponents are much larger than the near zero values expected and seen (Wolf et al. 1985) in periodic and quasi-periodic systems. The discrepancy between the theoretical and actual values is credited to the noise levels inherent in an open system. Deissler \& Kaneko (1987) suggests the use of a 'velocity-dependent Lyapunov exponent' in which the velocity fluctuations are measured in a moving frame of reference. However, the experimental implementation of this technique for wake flows has not been realized, particularly if one considers that a convective velocity $\left(\frac{1}{2}\left(U_{1}+U_{2}\right)\right.$ was suggested for mixing layers) is not readily determined for wake flows.

\subsection{The fuid dynamics of a chaotic wake}

The numerical diagnostics provide a measure of a system's divergence. Left unanswered is the question of the effects of chaos on flow parameters such as the vortex configuration, the mean and fluctuating velocities, and the drag.

\subsubsection{Flow visualization}

Flow visualization reveals the vortex configuration of the wake. A locked flow (figure 14) is characterized by the uniform staggered vortex pattern known as the Kármán vortex street. The second photo in the sequence (figure 15) presents a quasiperiodic flow which is identified by the existence of a beating pattern. The difference frequency is $0.625 \mathrm{~Hz}$. The next flow displayed (figure 16) is a quasi-periodic case in which the difference between the two frequencies is $1.4 \mathrm{~Hz}$. The wavelength of the almost identical clusters is decreased compared to the first quasi-periodic case. Within the clusters, pairing of the vortices is occurring. To complete the sequence, 

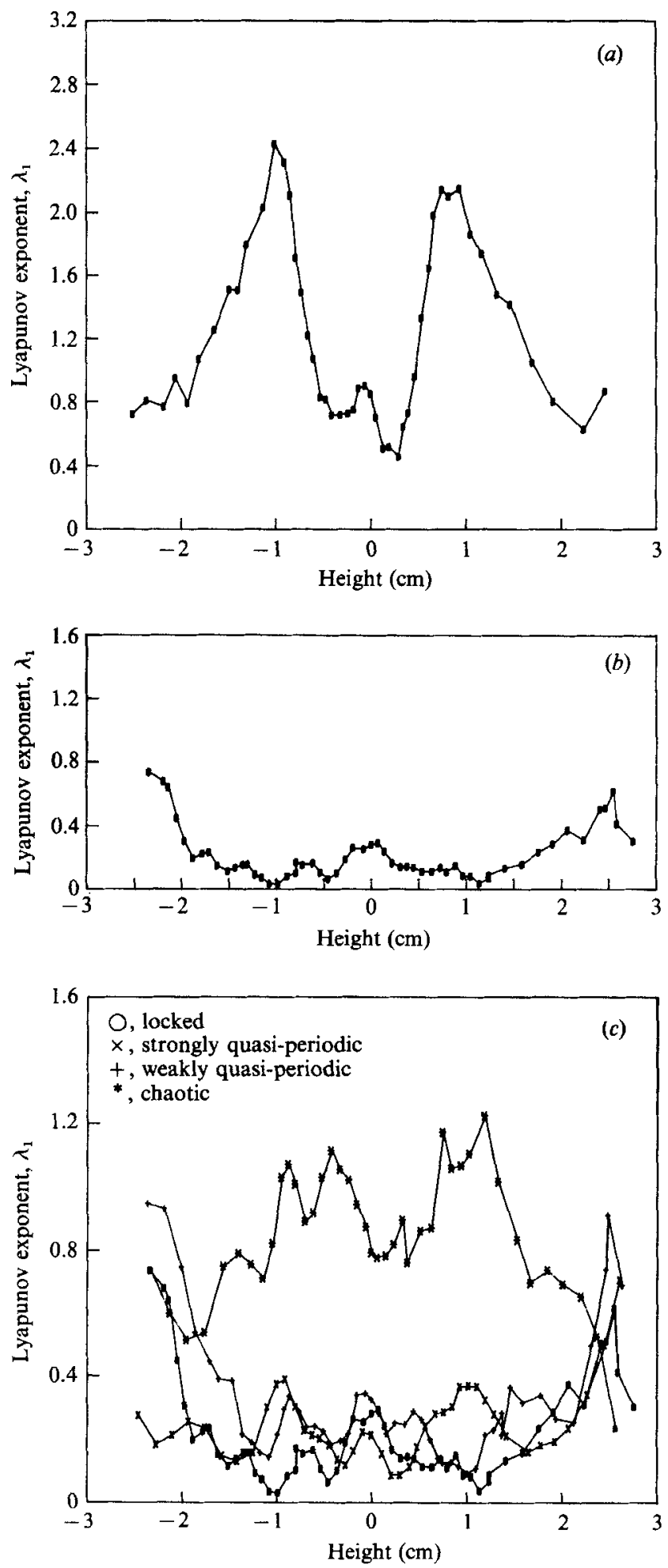

Figure 13. Lyapunov exponent as a function of height for $(a)$ the natural wake, $(b)$ the locked wake and $(c)$ the route to chaos cases (at $x / c=3$ ). 


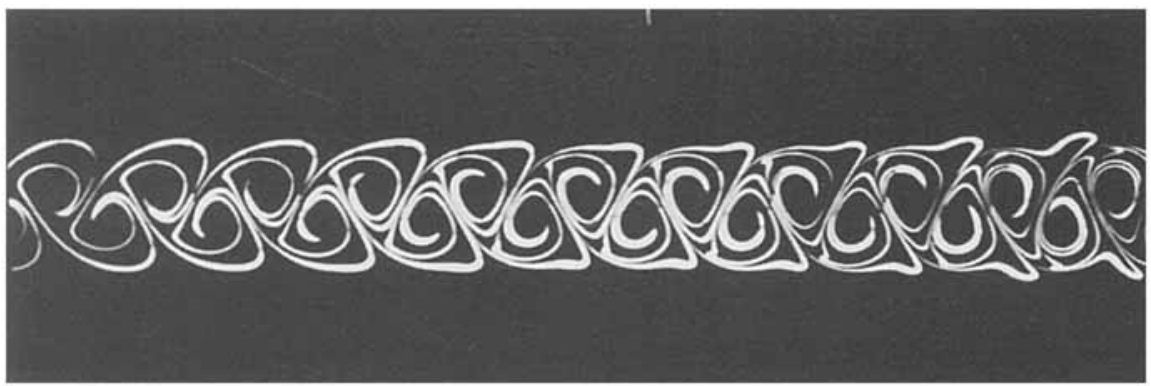

Figure 14. Flow visualization of locked flow: $f_{1}=4.075 \mathrm{~Hz}=$ natural vortex shedding frequency (flow is from left to right).

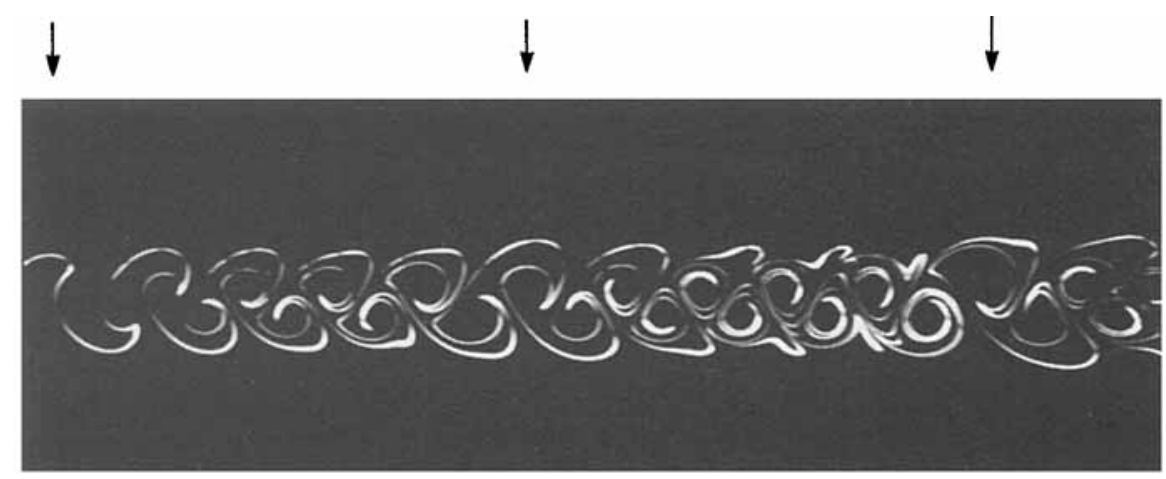

FIGURE 15. Flow visualization of quasi-periodic flow : $f_{1}=4.075 \mathrm{~Hz}=$ natural vortex shedding frequency, $f_{2}=4.7 \mathrm{~Hz}$ (flow is from left to right).

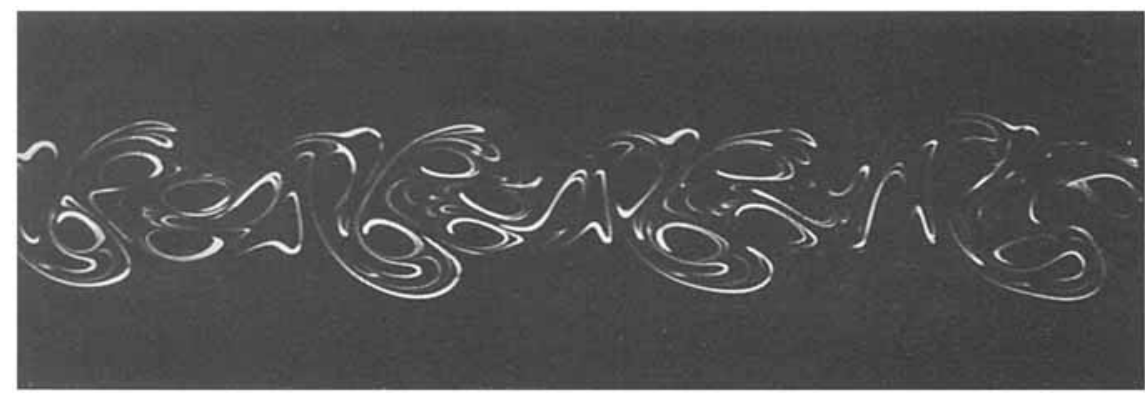

Figure 16. Flow visualization of quasi-periodic flow : $f_{1}=3.5 \mathrm{~Hz}, f_{2}=4.9 \mathrm{~Hz}$ (flow is from left to right).

examples of the chaotic case are presented in figure 17. A random nature to the vortex patterns exists. At times, segments of the Kármán street are visible; at other times, a great deal of stretching and diffusion is present. In light of the fact that patches of order exist, it can be said that the chaos is a result of the clash of orders rather than the lack of order.

\subsubsection{Velocity parameters and drag results}

Before considering entire velocity profiles, consider first a comparison between the probability density functions (PDF) of locked, strongly quasi-periodic, and chaotic 
(a)

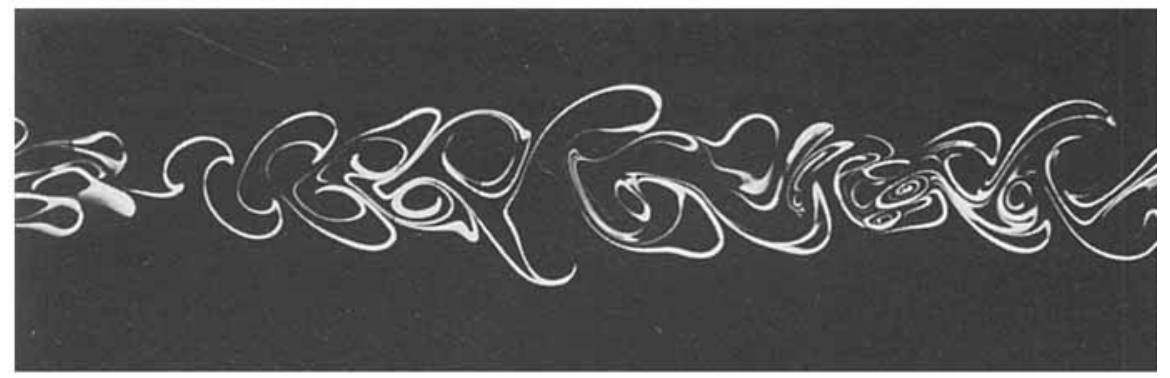

(b)

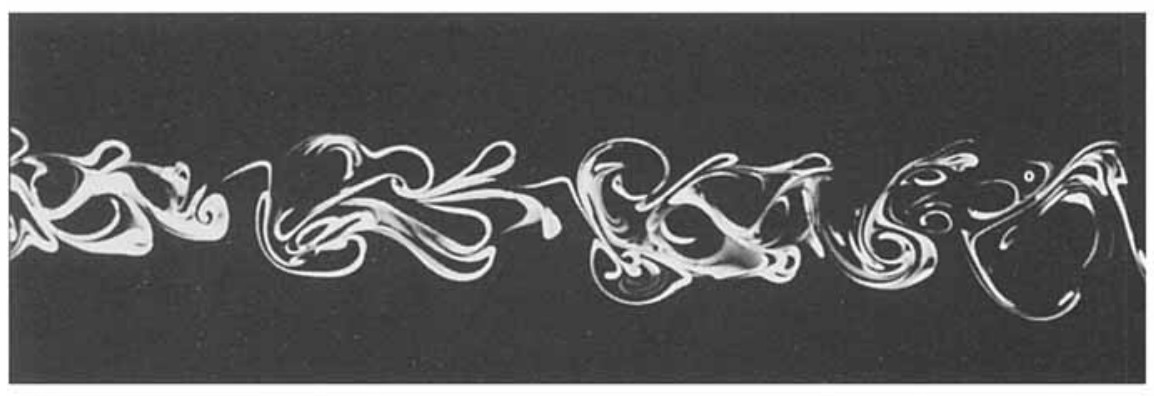

(c)

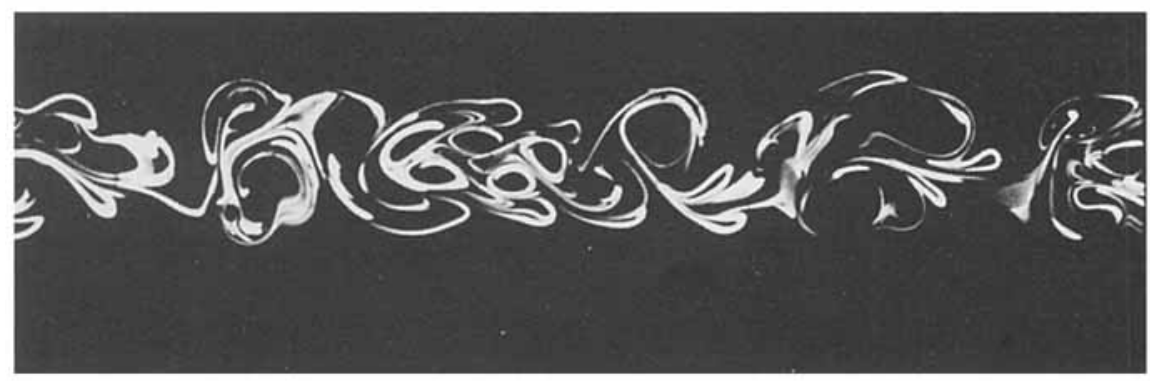

Figure $17(a-c)$. Flow visualization of chaotic flow: $f_{1}=3.55 \mathrm{~Hz}, f_{2}=5.71 \mathrm{~Hz}, f_{3}=f_{\mathrm{n}}$ (flow is from left to right).

time series (figure 18). The PDFs are calculated from data at $y=\delta$ in figure $18(a)$, and at $y=0$ in figure $18(b)$. The PDFs are presented such that

$$
\int_{-\infty}^{+\infty} p(u) \mathrm{d} u=1
$$

(where $u$ is the $x$-velocity). The ordered PDFs (top and middle) are characterized by the presence of two distinct peaks (similar to the PDF of a sine wave). The chaotic case, on the other hand, has PDFs which are approaching a Gaussian distribution.

A comparison between the autocorrelation functions of locked, strongly quasiperiodic, and chaotic time series (normalized by $\left.\left(u^{\prime 2}\right)^{\frac{1}{2}}\right)$ is presented in figure 19. The autocorrelations are calculated from data at $y=\delta$ in figure $19(a)$, and at $y=0$ in figure $19(b)$. The autocorrelation is useful in extracting deterministic data from a signal containing background (and stochastic) noise. The locked case autocorrelations 
(a)
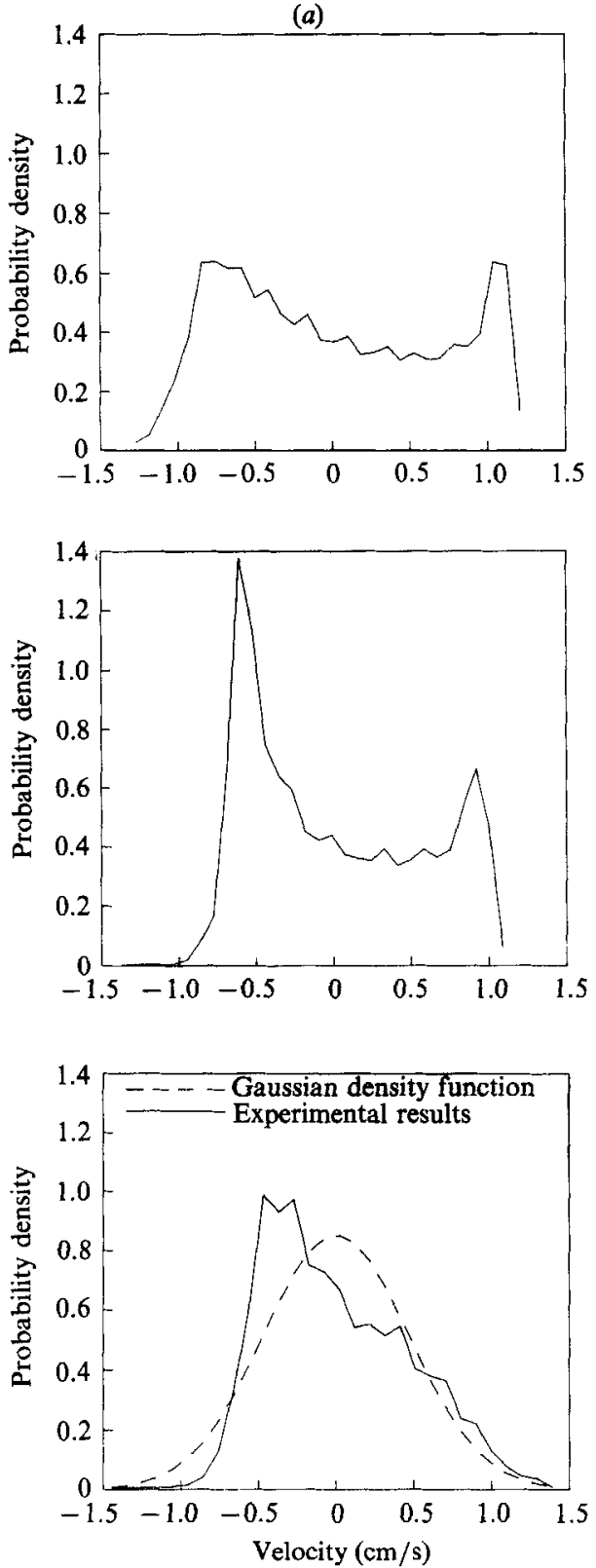

(b)
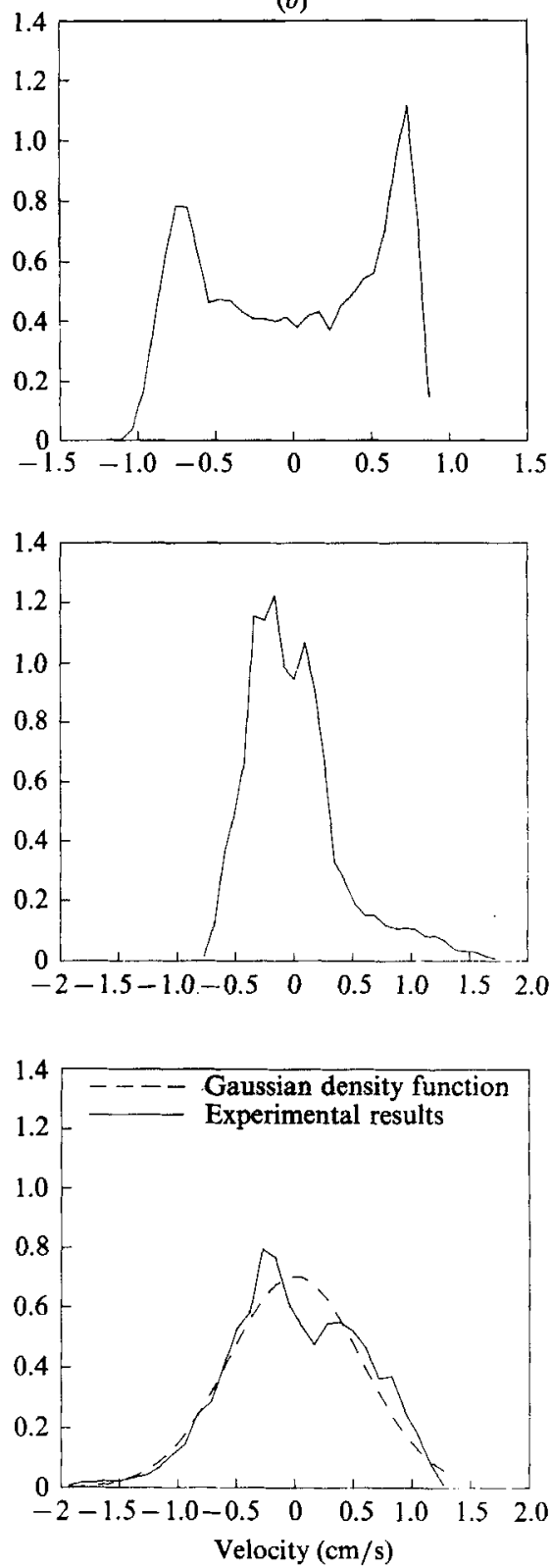

Figure 18. Probability density functions for route to chaos cases: $(a) y=\delta,(b) y=0$; top, locked; middle, quasi-periodic; bottom, chaotic.

(top in each figure) are characterized by a fairly uniform sine wave. The autocorrelations for a strongly quasi-periodic case (middle - difference frequency = $0.2 \mathrm{~Hz}$ ) contain a distinct beating indicative of the two frequencies in the system. For both of these ordered cases, the time series show strong correlation. The chaotic autocorrelations (bottom), on the other hand, are relatively uncorrelated, i.e. the system behaviour is random. The state of the system at time $t$ yields no insight as to its state at a later time $t+\tau$. 
(a)
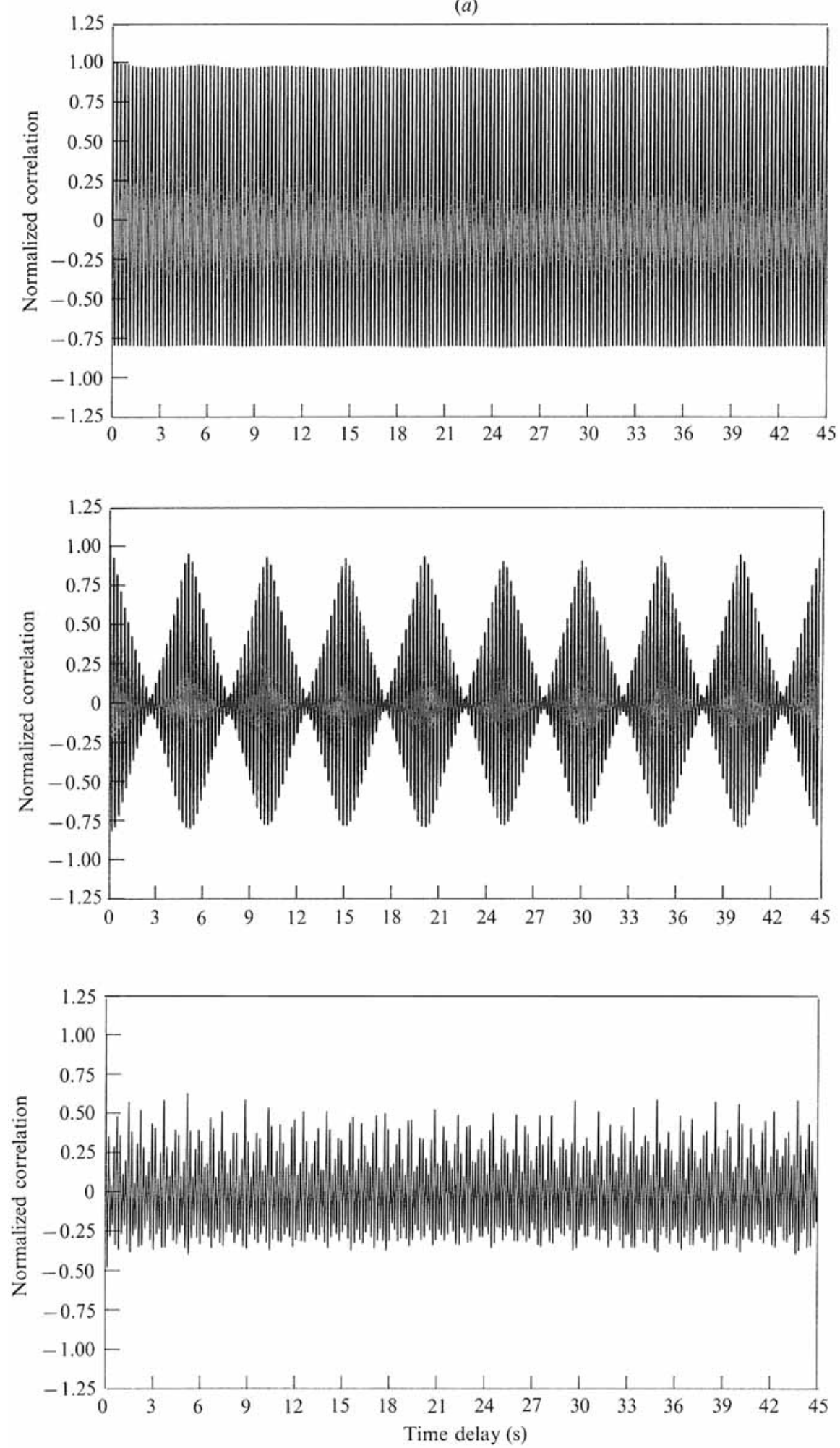

Figure $19(a)$. For caption see facing page. 

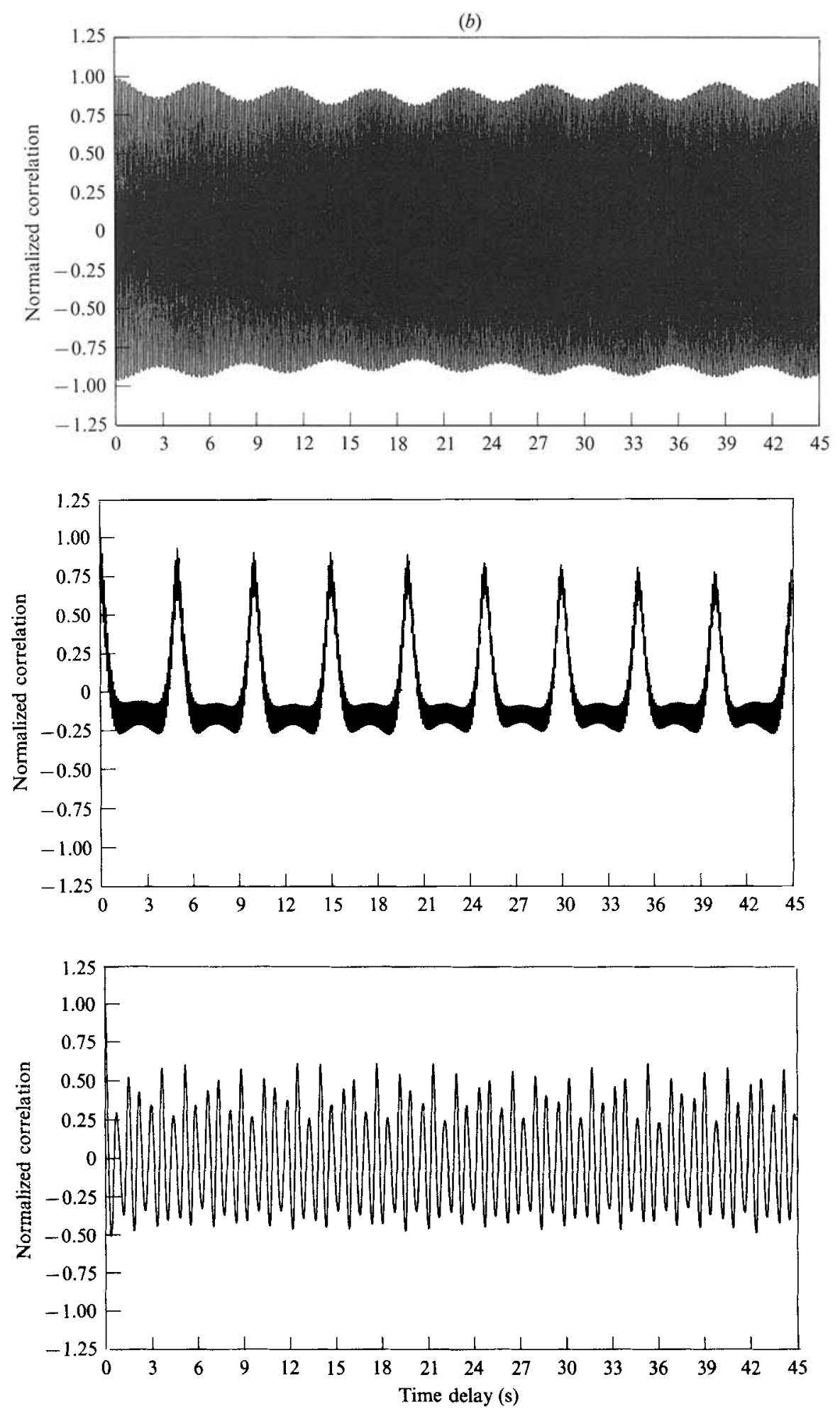

FigURE 19. Autocorrelation functions for route to chaos cases: $(a) y=\delta,(b) y=0$; top, locked; middle, quasi-periodic; bottom, chaotic. 

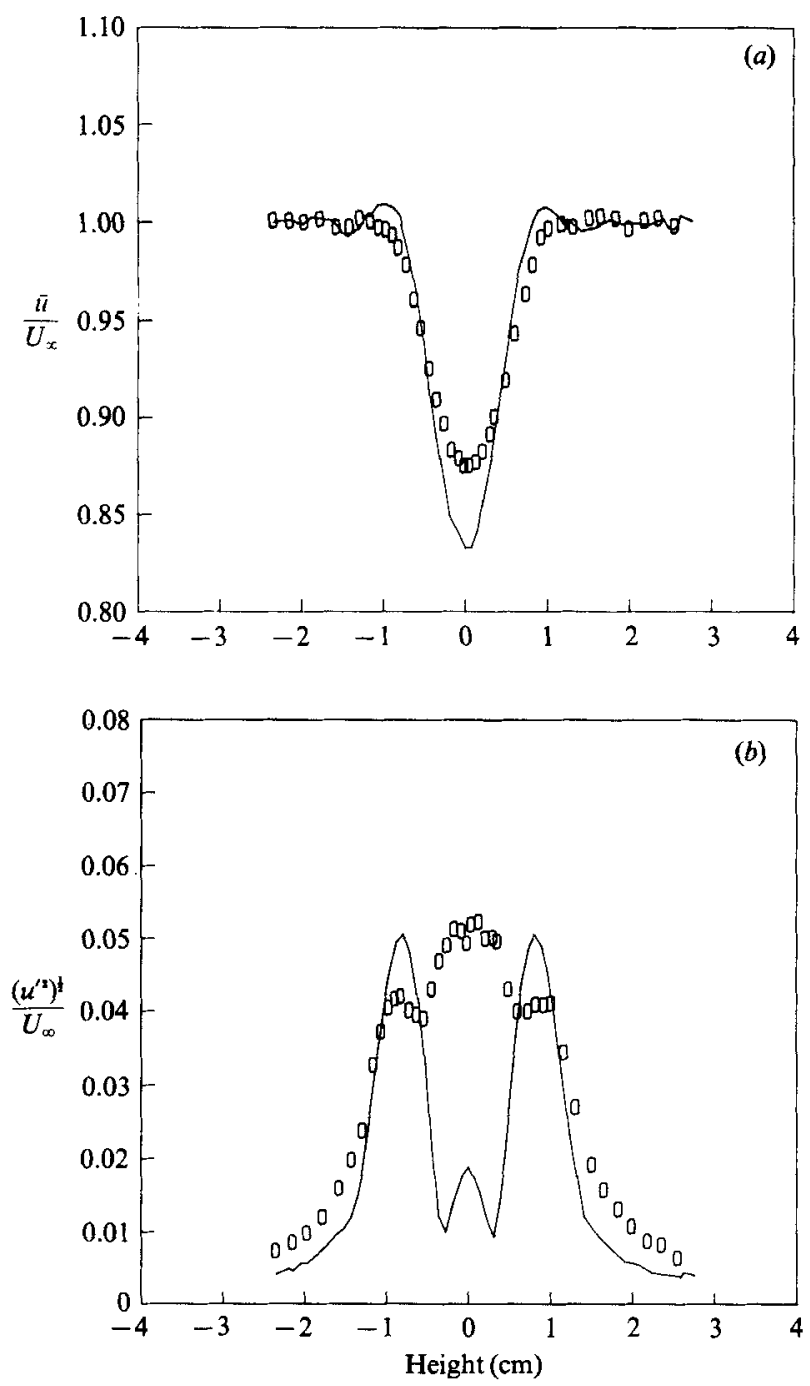

Figure 20. (a) Mean and (b) r.m.s. velocity profiles for the locked and chaotic cases (at $x / c=3$ ).

Consider next the velocity measurements. The mean and r.m.s. $\left(\left(u^{\prime 2}\right)^{\frac{1}{2}}\right)$ velocity profiles of the locked and chaotic flows are presented in figure 20. The locked case is indicated by a solid line, the chaotic case by the circles. In examining the mean velocity profile, the chaotic case has a smaller deficit compared to the ordered case. A more dramatic difference between the ordered and chaotic cases appears in the r.m.s. profiles. The chaotic case contains a third peak (an overshoot) on the centreline of the wake. A similar overshoot in the r.m.s. profile was observed by Sato \& Kuriki (1961) for the transition of a natural wake of a thin flat plate, and by Sato (1970) for a two-frequency forced wake. The overshoot was attributed, in the first paper, to the development of fluctuations at the second harmonic of the natural shedding frequency and, in the latter paper, to fluctuations at the difference between the two forcing frequencies.

To further explore possible sources of the third peak in the chaotic airfoil wake, 

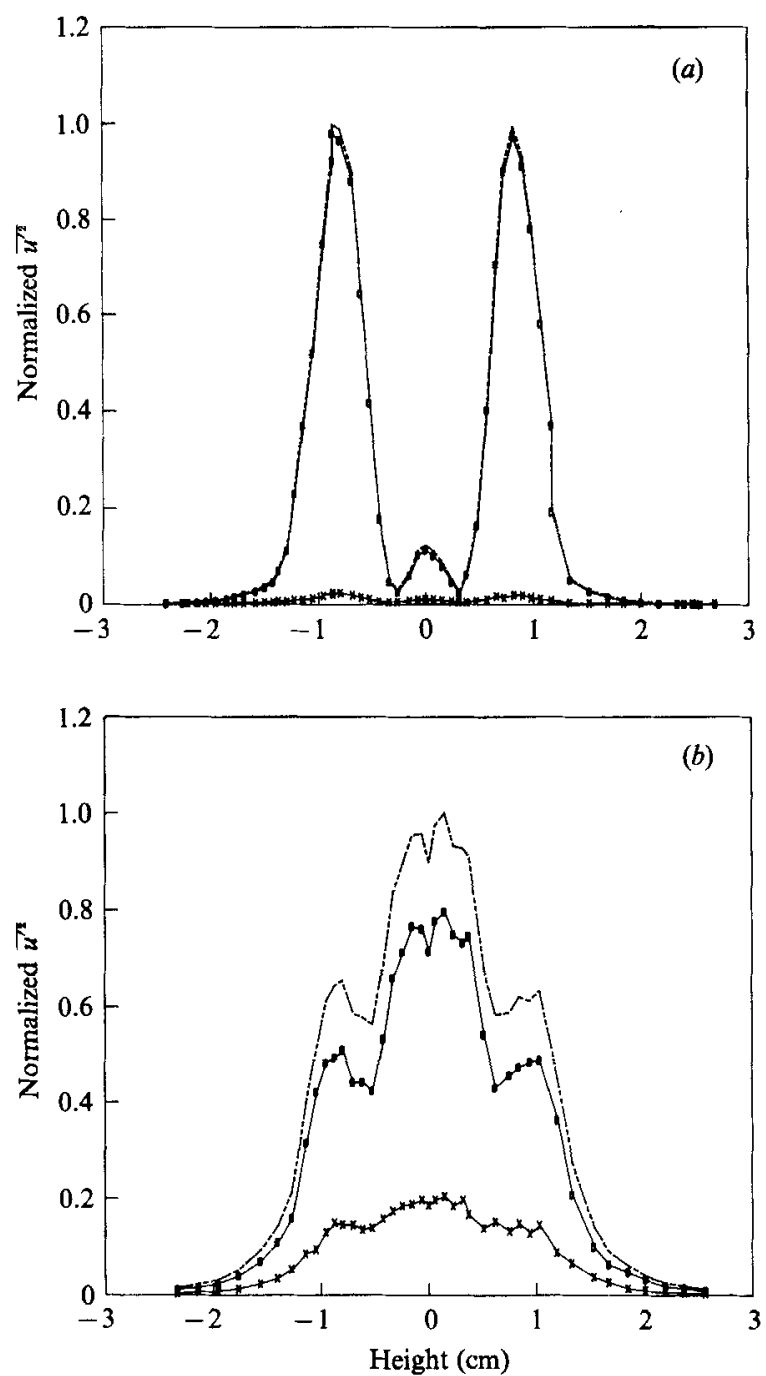

FiguRe 21. Bandwidth $\overline{u^{i 2}}$ profile showing the contribution to the broadband $\overline{u^{\prime 2}}$ profile of all distinct frequencies versus the contribution of random noise for $(a)$ the locked case and $(b)$ the chaotic case (at $x / c=3$ ). $\bigcirc$, Distinct frequencies; $\mathrm{x}$, noise.

bandwidth $\overline{u^{\prime 2}}$ profiles were constructed by considering contributions to the fluctuations due to all of the distinct frequencies in the flow (either the forcing frequencies or their linear combinations) and then comparing the results to the contributions due to random noise (i.e. the remainder of the area under the power spectrum after the distinct frequencies were removed). The results are presented in figure 21 . The total $\overline{u^{\prime 2}}$ is indicated with a dashed line. The contribution from distinct frequencies is indicated with a circle and from random noise with a cross. For the locked case (figure $21 a$ ) the majority of the fluctuations can be attributed to the forcing frequency and its harmonics. In contrast, for the chaotic case (figure $21 b$ ), distinct frequencies are only partial contributors to the overall fluctuations. A significant level of fluctuations can be attributed to noise, particularly near the centre of the wake. 


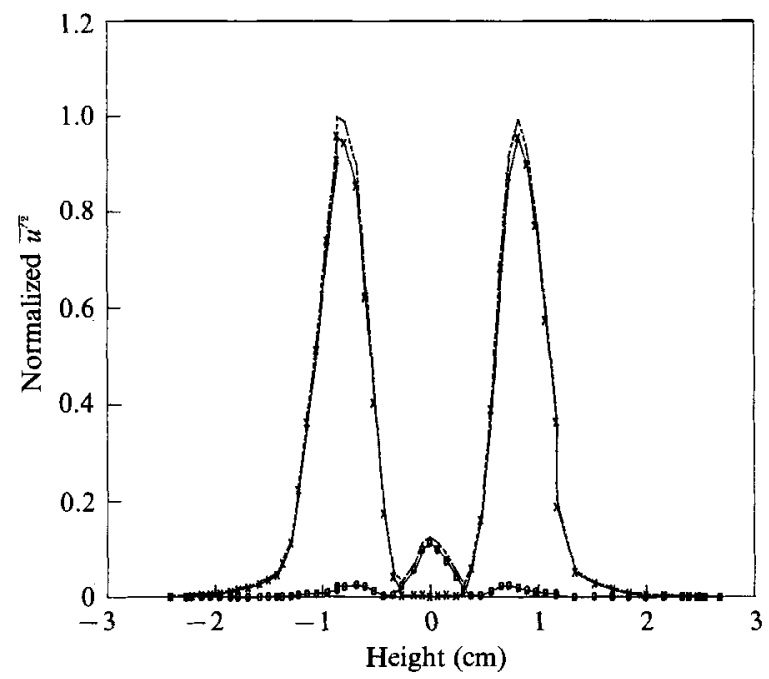

Figure 22. Bandwidth $\overline{u^{\prime 2}}$ profile of the locked case showing the contribution of the primary frequency $(x)$ and its second harmonic $(0)($ at $x / c=3$ ).

In order to better understand the nature of fluctuations due to distinct frequencies (i.e. which frequencies contribute to which peaks), more bandwidth profiles were created. For the locked case (figure 22), the contribution of the natural frequency and its second harmonic are compared to the overall fluctuations (the dashed line). The natural frequency is the primary source of fluctuations for the two side peaks. The second harmonic is the main source of fluctuations on the centreline. This observation is consistent with the findings of other wake investigators (Sato \& Kuriki 1961; and Wygnanski, Champagne \& Marasli 1986).

With the results of Sato's two-frequency forcing in mind, the contributions to $\overline{u^{\prime 2}}$ from the top and bottom forcing frequencies, the natural frequency, and the difference frequencies $\left|f_{2}-f_{1}\right|,\left|f_{2}-f_{\mathrm{n}}\right|$, and $\left|f_{\mathrm{n}}-f_{1}\right|$ were examined for the chaotic case (figure 23). Like the locked case, the chaotic case shows contributions of the three primary frequencies to the two side lobes (figure $23 a$ ). In contrast, the main components of the centreline fluctuations (figure $23 b$ ) are the interaction frequencies $-\left|f_{2}-f_{1}\right|,\left|f_{2}-f_{\mathrm{n}}\right|$, and $\left|f_{\mathrm{n}}-f_{1}\right|$. The second harmonics of the primary frequencies are negligible contributors to the flow at any position across the chaotic wake. In summary, the transition from locked to chaotic flow corresponds to a transition in the fluctuation profiles. The second harmonic is the dominant centreline frequency for the locked case. At the end of the transition, the interaction frequencies characterize the centreline fluctuations.

The increased fluctuations at the centreline and the smaller wake deficit for the chaotic case would seem to imply a reduction in drag. However, the profiles presented are measured at $x / c=3$. In $\mathrm{I}$, it was mentioned that, at $x / c=3$, contributions from the $y$-component of velocity become significant. To determine the drag coefficient of the locked and chaotic cases, a control-volume momentum analysis was applied to velocity profiles measured at $x / c=1$ (where the assumption of $V=0$ is valid). The drag coefficient for the locked case revealed a reduction of drag by $15 \%$ relative to the natural case. Similar drag results were seen for locked cases in I The chaotic case resulted in a $3 \%$ increase in drag when compared to the natural case. This is a relatively insignificant change. 

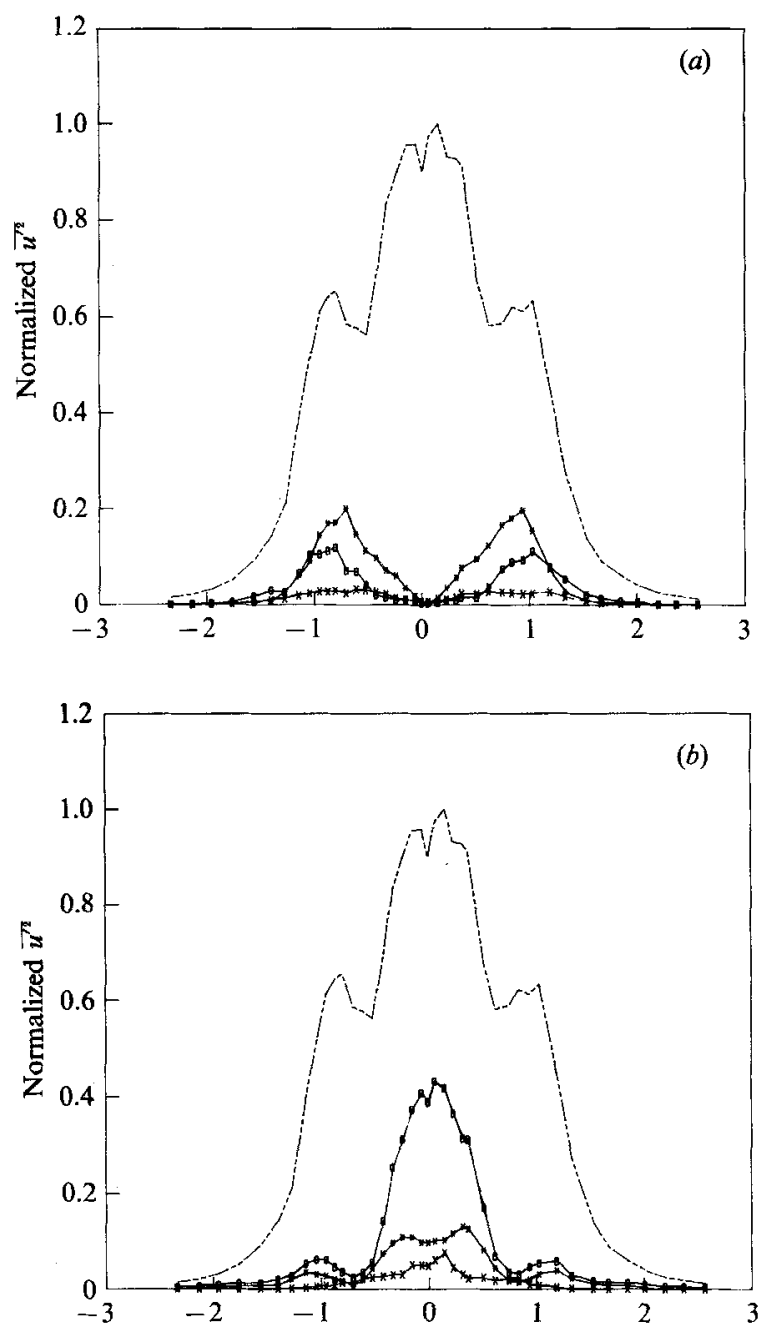

Figure 23. Bandwidth $\overline{u^{\prime 2}}$ profile of the chaotic case showing the contribution of $(a) f_{2}(\times), f_{2}$ $(*)$, and $f_{\mathrm{n}}(O) ;(b)\left|f_{2}-f_{1}\right|(*),\left|f_{2}-f_{\mathrm{n}}\right|(O)$, and $\left|f_{\mathrm{n}}-f_{1}\right|,(\times)$.

\subsection{Chaos and turbulence - are they related?}

When examining the flow visualization for the chaotic case, a range of scales of motion is apparent, and some areas with strong mixing and dissipation exist. For the chaotic case, it was observed that three-dimensional motion (out of the picture plane) existed which was not present in the locked case. If one attempts to define turbulence by defining its characteristics, in a manner similar to Tennekes \& Lumley (1972), (irregularity (randomness), three-dimensional vorticity fluctuations, rapid mixing/diffusion, and strong dissipation), it appears that the chaotic case contains regions which are locally turbulent. An attempt is made here to determine statistically whether the laminar chaotic case is similar to a high Reynolds number turbulent flow. As was already seen in the presentation of the velocity data, the probability density functions of the time series for the chaotic case are slightly Gaussian shaped and the autocorrelation functions are fairly uncorrelated (both functions indicating a state of randomness). Using the wavenumber spectrum as a diagnostic, a comparison 


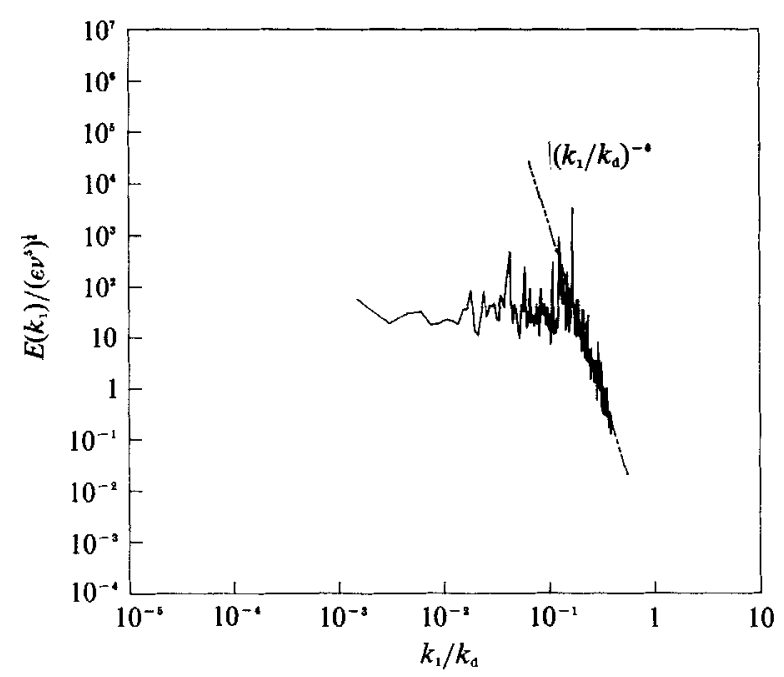

Figure 24. Energy spectrum of chaotic case at $y=\delta$ (at $x / c=3$ ).

of the chaotic spectrum to Kolmogorov's ' $k$ to the $-\frac{5}{3}$ ' law is performed. For isotropic turbulence in the inertial subrange, the wavenumber magnitude spectral density $E(k)$ can be related to the wavenumber $k$ by $E(k) \propto k^{-\frac{5}{3}}$. The quantity measured in the experiment is the Fourier power spectrum $\phi(\omega)$ where $\omega$ is radian frequency. Bradshaw (1971) indicates that for most purposes $\phi(\omega)$ can be used as an approximation to $\phi\left(k_{1}\right)$ which in turn is proportional to $E(k) . k_{1}$ is the wavenumber in the downstream direction of the flow. The one-dimensional energy spectrum, $E\left(k_{1}\right)$, is presented in non-dimensional form (scaled by $\left.\left(\epsilon \nu^{5}\right)^{\frac{1}{4}}\right)$ as a function of nondimensional wavenumber $\left(k_{1} / k_{\mathrm{d}}\right)$, where $k_{\mathrm{d}}$ is the wavenumber associated with the Kolmogorov lengthscale $(\eta)$. The dissipation $\epsilon$ was determined from a numerical approximation

$$
\epsilon=15 \nu \int_{-\infty}^{+\infty} k^{2} E(k) \mathrm{d} k .
$$

Additional estimates for $\epsilon$ were performed with relatively little change to the results.

The wavenumber spectra for the chaotic case at $y=\delta$ and at the centreline are shown in figures 24 and 25 with a log-log scaling. The results at $y=\delta$ show a -6 slope over less than a decade. In contrast, the centreline spectrum results are very close to a $-\frac{5}{3}$ slope over a wider range of wavenumbers. An analytical curve by Pao (1965) and some experimental data points for low Reynolds number $\left(R e_{\lambda} \approx 30\right)$ turbulent flows of Uberoi \& Freymuth (1969) and Comte-Bellot \& Corrsin (1971) are plotted on the centreline spectrum. There is a qualitative agreement between the chaotic case and the turbulent cases. The spectrum results indicate that a local behaviour exists which shares the $-\frac{5}{3}$ decay characteristic of a fully turbulent flow. On one hand, this is quite surprising when one remembers that the flow is a low Reynolds number $\left(\mathrm{Re}_{\delta}\right.$ ) 'laminar' flow and that the flow is far from isotropic.

However, when scaled by the viscous dissipation, $\epsilon_{v}=v\left(u / l_{\mathrm{e}}\right)^{2}\left(l_{\mathrm{e}}\right.$ being a typical eddy size), the energy spectrum is in better quantitative agreement with the turbulent data (figure 26). This observation implies that the turbulent dissipation due to Reynolds stresses is still small compared to the viscous dissipation of the large-scale eddies. 


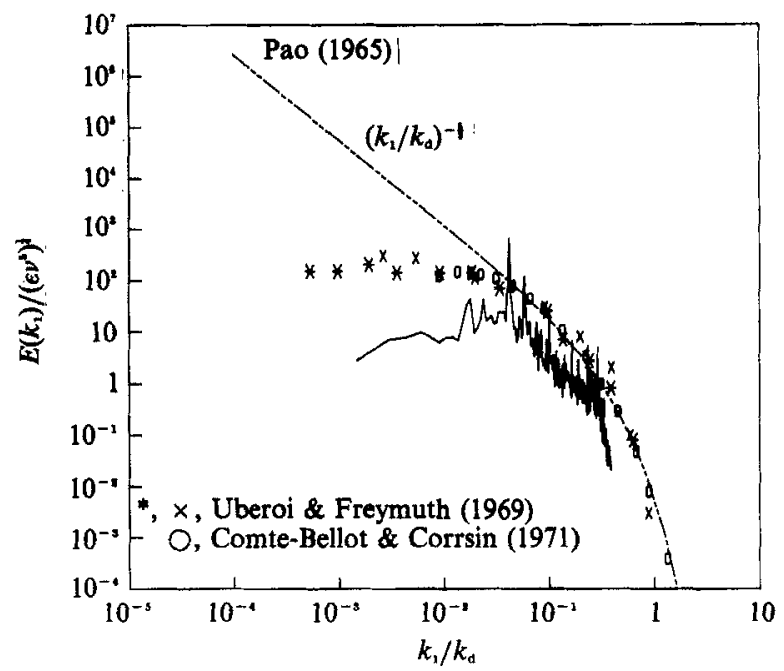

FIGURE 25. Energy spectrum of chaotic case at $y=0$ (at $x / c=3$ ) (dissipation calculated from $\left.\varepsilon=15 v \int k^{2} E(k) \mathrm{d} k\right)$.

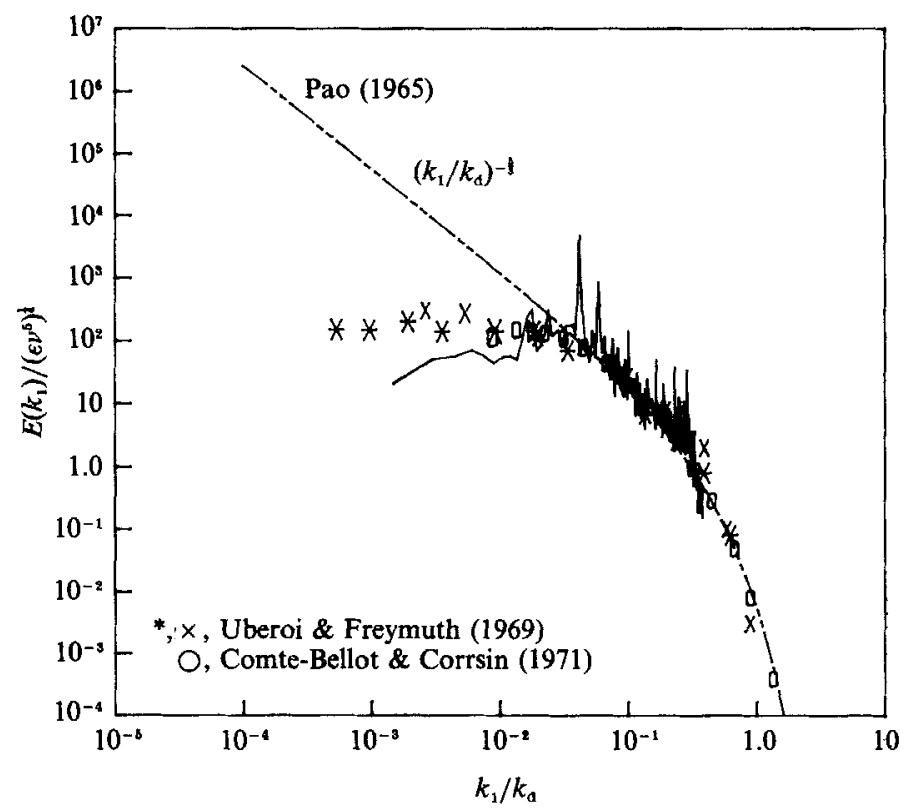

FigURE 26. Energy spectrum of chaotic case at $y=0$ (at $x / c=3$ ) (dissipation calculated using viscous form $\left.\epsilon_{\nu}=\nu\left(u / l_{\mathrm{e}}\right)^{2}\right)$.

It is noted that the application of a ' $-\frac{5}{3}$ analysis' for a flow with a low Reynolds number is on the borderline of acceptability. (The criterion for the universal equilibrium range (Hinze $1975, l_{\mathrm{e}} \gg \eta$ ) is barely satisfied.) However, at the same time the appearance of the $-\frac{5}{3}$ behaviour at the centreline (before other regions of the wake) is consistent with the observations of Sato \& Kuriki on the natural transition to turbulence in the wake of the flat plate. In addition, one would expect to find the most rapid transition at the point in the wake where the maximum interaction 


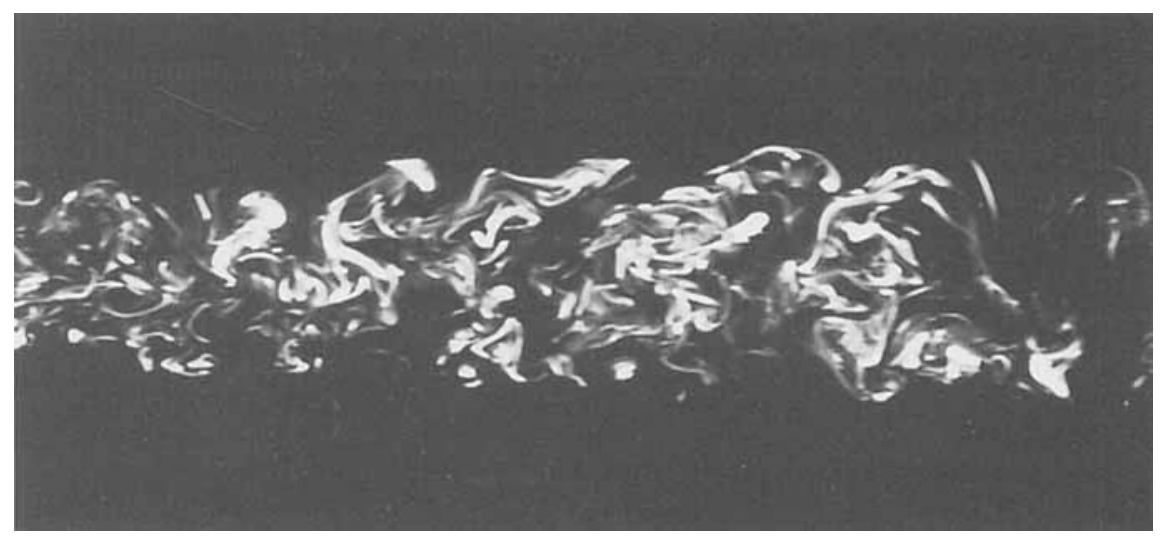

FigURe 27. Flow visualization of a turbulent wake $\left(U_{\infty}=13 \mathrm{~cm} / \mathrm{s}\right)$.

between competing modes occurs, which is along the centreline for the forced wake. The -6 decay is possibly an artifact of instrument response which is overwhelmed by the $-\frac{5}{3}$ behaviour at the centreline of the chaotic case.

The competition of multiple modes is presented as a major stepping stone along the route to turbulent flow. To provide the reader with a visual means of comparison between chaos and turbulence, and to reinforce the concept that the chaotic flow is not globally turbulent, flow visualization of a turbulent wake is presented in figure 27. The turbulent wake was created by tripping the airfoil boundary layers and increasing the mean flow velocity to $13 \mathrm{~cm} / \mathrm{s}$. The resulting turbulent flow is characterized by the presence of a more uniform range of scales when compared to the chaotic case.

\section{Concluding remarks}

For a long time, the phenomenon of chaos in open fluid systems has been synonymous with turbulence. It is presumed that turbulence/chaos in wakes occurs when the Reynolds number is relatively large. However, in this paper it is seen that a chaotic state is achieved at low Reynolds number as a result of the nonlinear interaction of three incommensurate frequencies in the wake system. This chaotic state shares some characteristics with turbulent flows, but is not globally turbulent. This is because the chaos is generated by the competition of local order. The level of competition determines the level of chaos, which in its highest state represents turbulence. A noteworthy point is that the individual frequencies when input alone establish order in the system, yet when input simultaneously generate randomness. With this in mind, the implications are considerable for those researchers who are modelling the laminar/turbulent transition process or are applying these models to achieve improved aerodynamic performance through feedback control.

The application of diagnostic tools from the field of nonlinear dynamics provides qualitative insight into the nature of the wake system. However, quantitative results similar to those seen for experiments on closed systems are not obtainable, owing in part to the high level of background noise associated with open flows. A need is seen for the development of more effective dynamical system diagnostic techniques for open systems. In particular, multi-point measurements (from both an Eulerian and Lagrangian perspective) would be better suited for open flows. 
This paper is based on the work from the Ph.D. thesis of K. Stuber which was carried out at the University of California, San Diego. The work was sponsored by the Defense Advanced Research Projects Agency - ACMP through the URI program under contract number DARPA/ONR N00014-86-K-0758. The assistance of INLS/UCSD is also acknowledged. The authors would like to thank Professor M. Mârkovin with whom we had worthwhile discussions regarding the nature of chaos and turbulence.

\section{REFERENCES}

Bradshaw, P. 1971 An Introduction to Turbulence and its Measurement. Pergamon.

Comte-Bellot, G. \& Corrsin, S. 1971 Simple Eulerian time correlation of full- and narrow-band velocity signals in grid-generated, 'isotropic' turbulence. J. Fluid Mech. 48, 273.

Deissler, R. J. \& Kaneko. K. 1987 Velocity-dependent Lyapunov exponents as a measure of chaos for open-flow systems. Phys. Lett. $A$ 119, 397.

Eckmann, J.-P. \& Ruelle, D. 1985 Ergodic theory of chaos and strange attractors. Rev. Mod. Phys. 57, 617.

Farmer, J. D., Ott, E. \& Yorke, J. A. 1983 The dimension of Chaotic Attractors. Physica 7D, 153.

Gharib, M. \& Williams-Stuber, K. 1989 Experiments on the forced wake of an airfoil. $J$. Fluid Mech. 208, 225 (referred to as I).

Gollub, J. P. \& Benson, S. V. 1980 Many routes to turbulent convection. J. Fluid Mech. 100, 449.

Hinze, J. O. 1975 Turbulence. McGraw-Hill.

Kolmogorov, A. N. 1941 The local structure of turbulence in incompressible viscous fluid for very large Reynolds numbers. C.R. Acad. Sci. URSS 30, 301.

Newhouse, S., Ruelle, D. \& Takens, F. 1978 Occurrence of strange axiom A attractors near quasi periodic flows on $T^{m}, m \geqslant 3$. Commun. Math. Phys. 64, 35 .

Oseledec, V. J. 1968 A multiplicative ergodic theorem. Lyapunov characteristic numbers for dynamical systems. Trans. Moscow Math. Soc. 519, 197.

Packard, N. H., Crutchfield, J. P., Farmer, J. D. \& Shaw, R. S. 1980 Geometry from time series. Phys. Rev. Lett. 45, 712.

PAO, Y.-H. 1965 Structure of turbulent velocity and scalar fields at large wavenumbers. Phys. Fluids 8, 1063.

SATO, H. 1970 An experimental study of non-linear interaction of velocity fluctuations in the transition region of a two-dimensional wake. J. Fluid Mech. 44, 741.

Sato, H. \& KurIKI, K. 1961 The mechanism of transition in the wake of a thin flat plate placed parallel to uniform flow. J. Fluid Mech. 11, 321.

SReenivasan, K. R, 1985 Transition and turbulence in fluid flows and low dimensional chaos. In Frontiers in Fluid Mechanics (ed. S. H. Davis \& J. L. Lumley), pp. 41-67. Springer.

STUBER, K. 1988 An experimental investigation of the effect of external forcing on the wake of a thin airfoil. Ph.D. thesis, University of California, San Diego, La Jolla, CA.

Swinney, H. L. 1983 Observations of order and chaos in nonlinear systems. Physica 7D, 3.

TAKENS, F. 1981 Detecting strange attractors in turbulence. In Dynamical Systems \& Turbulence, Warwick 1980. Lecture Notes in Mathematics, vol. 898, p. 366. Springer.

Tennekes, H. \& Lumley, J. L. 1972 A First Course in Turbulence. The MIT Press.

Uberoi, M. S. \& Freymuth, P. 1969 Spectra of turbulence in wakes behind circular cylinders. Phys. Fluids 12, 1359.

VAn AtTa, C. W. \& Gharib, M. 1987 Ordered and chaotic vortex streets behind circular cylinders at low Reynolds numbers. J. Fluid Mech. 174, 113.

Wolf, A., Swift, J. B., Swinney, H. L. \& Vastano, J.A. 1985 Determining Lyapunov exponents from a time series. Physica 16D, 285.

Wyenanski, I., Champagne, F. \& Marasli, B. $1986^{\circ}$ On the large-scale structures in twodimensional, small-deficit, turbulent wakes. J. Fluid Mech. 168, 31. 